COMMISSIONING OF A VNA DYNAMIC UNCERTAINTY TOOL FOR MICROWAVE S-PARAMETER MEASUREMENTS

DILBAGH SINGH, MARTIN SALTER, JAMES SKINNER AND NICK RIDLER

JANUARY 2021 
NPL Report TQE 16 


\title{
COMMISSIONING OF A VNA DYNAMIC UNCERTAINTY TOOL FOR MICROWAVE S-PARAMETER MEASUREMENTS
}

\author{
D Singh, M J Salter J Skinner and N M Ridler \\ Electromagnetic \& Electrochemical Technologies Department
}

\begin{abstract}
All measurements have an associated uncertainty and it is important to know the uncertainty in order to quantify the reliability of the measurement result. At the National Physical Laboratory (NPL), the provision of scattering parameter (S-parameter) measurements at microwave frequencies for customers is achieved using the Primary Impedance Microwave Measurement System (PIMMS). PIMMS also evaluates the uncertainty in the measurements. The use of PIMMS can be quite onerous as several repeat calibrations and measurements are required to evaluate the measurement uncertainty, which is calculated during post processing of the measured data.

The Vector Network Analyser Dynamic Uncertainty Option (VNA-DUO) is available on PNA and PNA-X network analysers from Keysight Technologies and enables the real time display of S-parameter measurements and the associated measurement uncertainty. The uncertainties are calculated within the VNA and are displayed on the screen as error bars in real time.

This report describes the commissioning of VNA-DUO at NPL by measuring the S-parameters of several one- and two-port devices using both VNA-DUO and PIMMS, and the results from the two methods are compared. The report discusses the components of uncertainty that contribute to the overall uncertainty in the S-parameter measurements made using VNA-DUO and investigates the influence of each component on the overall uncertainty. Thru Reflect Line (TRL) calibration, Short Open Load Reciprocal (SOLR) calibration and an Electronic Calibration (ECal) module have been used for the VNA-DUO measurements. TRL calibration was used for the PIMMS measurements. The investigation was carried out in $3.5 \mathrm{~mm}$ coaxial line over the frequency range $100 \mathrm{MHz}$ to $26 \mathrm{GHz}$. To obtain a wide range of measurement values, one-port devices with very low to very high return loss and two-port devices with very low to very high insertion loss have been used.
\end{abstract}


(C) NPL Management Limited, 2021

\author{
ISSN 1754-2995 \\ https://doi.org/10.47120/npl.TQE16 \\ National Physical Laboratory \\ Hampton Road, Teddington, Middlesex, TW11 OLW
}

Extracts from this report may be reproduced provided the source is acknowledged and the extract is not taken out of context.

Approved on behalf of NPLML by

Michelle Apostolou, Head of Department, Electromagnetic \& Electrochemical Technologies Department 


\section{CONTENTS}

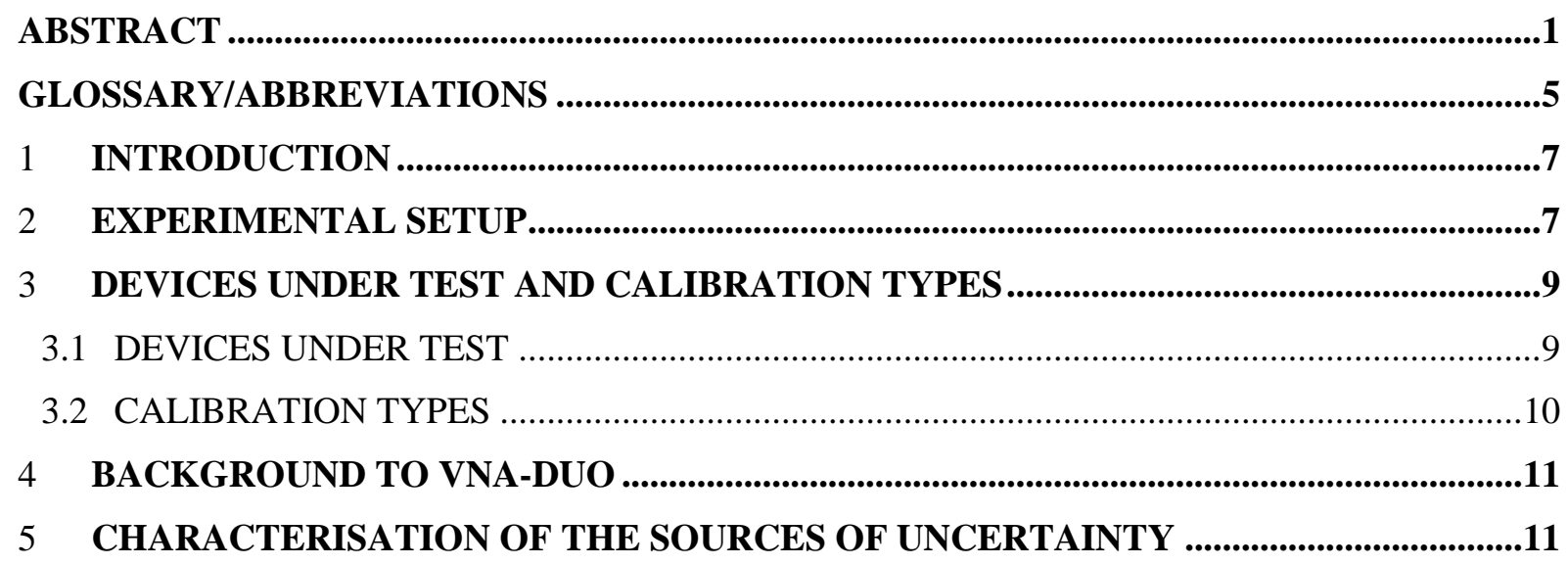

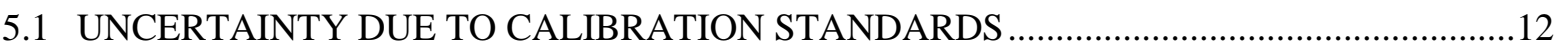

5.1.1 Calibration standard definitions supplied by the manufacturer ......................................12

5.1.2 Calibration standard definitions based on mechanical dimensions .................................12

5.1.3 Calibration standard definitions based on PIMMS measurements ....................................12

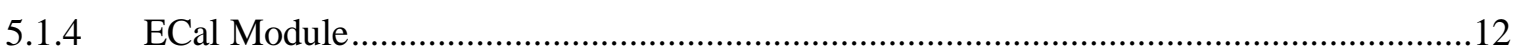

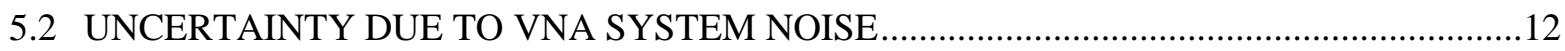

5.3 UNCERTAINTY DUE TO CONNECTOR AND CABLE REPEATABILITY ..........................12

6 COMPARISON OF THREE DIFFERENT METHODS FOR CHARACTERISING THE

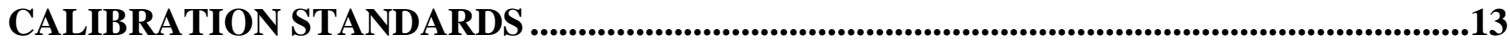

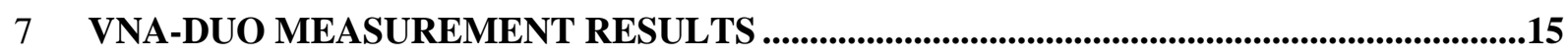

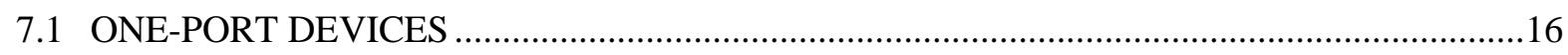

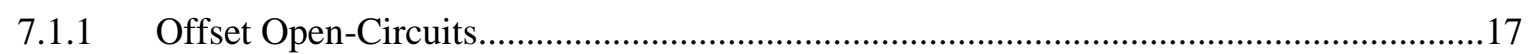

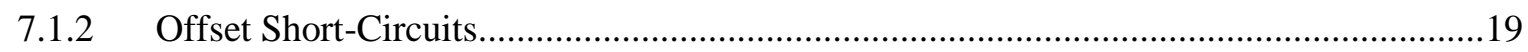

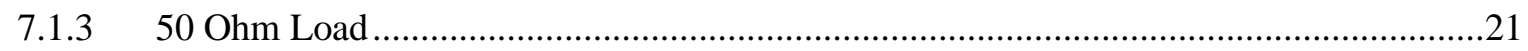

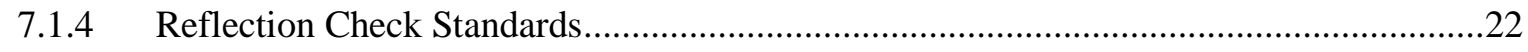

7.1.5 Summary of One-Port Device Measurements ................................................................

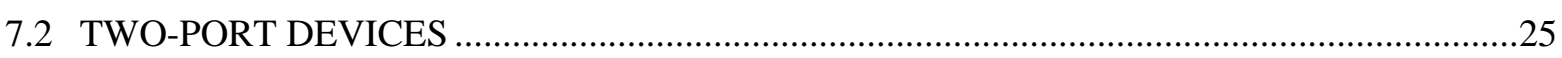

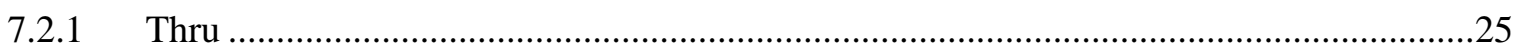

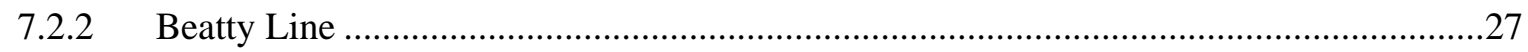

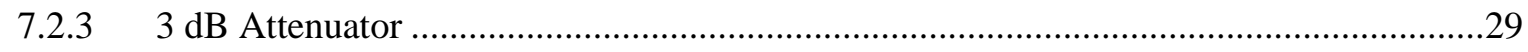

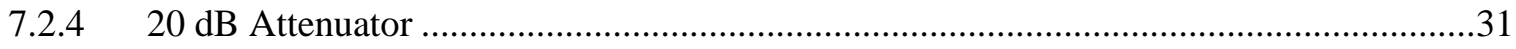

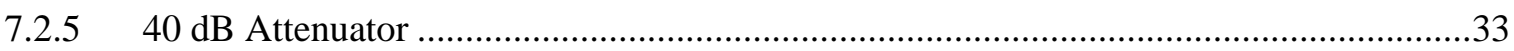

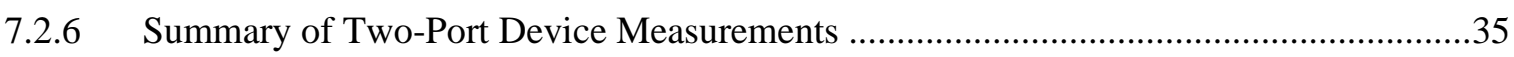

8 INVESTIGATION OF DIFFERENT SOURCES OF UNCERTAINTY ..................................37

9 CONTRIBUTION OF EACH CALIBRATION STANDARD TO THE MEASUREMENT

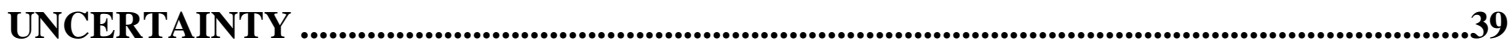


NPL Report TQE 16

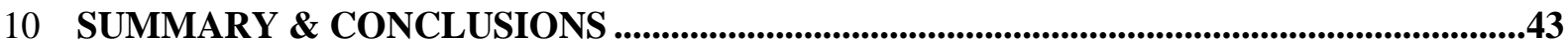

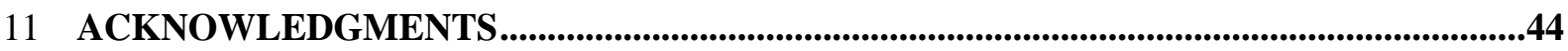

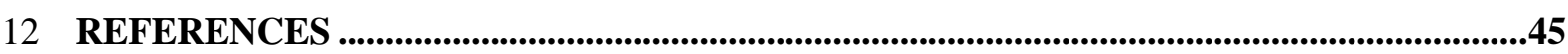

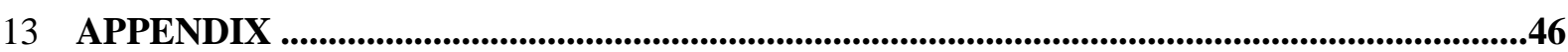




\section{GLOSSARY/ABBREVIATIONS}

DUT Device Under Test

ECal An electronic calibration module used to calibrate a VNA

$\mathrm{F} \quad$ Used to indicate a female coaxial connector

IF Intermediate frequency

IFBW IF bandwidth

LO Local oscillator

M Used to indicate a male coaxial connector

mU Milliunit

NER Normalised Error Ratio

NIST National Institute of Standards and Technology

NMI National Metrology Institute

NPL National Physical Laboratory

PIMMS NPL's Primary Impedance Microwave Measurement System

PTB Physikalisch-Technische Bundesanstalt

QSOLT Quick-Short-Open-Load-Thru method of VNA calibration

RF Radio frequency

SOLR Short Open Load Reciprocal method of VNA calibration (also known as the Unknown Thru method)

SOLT Short Open Load Thru method of VNA calibration

S-parameters Scattering parameters of a high frequency device or component

TRL Thru Reflect Line method of VNA calibration

VNA Vector Network Analyser

VNA-DUO VNA Dynamic Uncertainty Option available from Keysight Technologies for PNA and PNA-X VNAs. It is also known as "Option 015" and "S93015A". 
NPL Report TQE 16 


\section{INTRODUCTION}

The performance of a high frequency device or component is usually characterised using a Vector Network Analyser (VNA) which measures the complex-valued scattering parameters (or S-parameters) of the component -i.e. the magnitudes and phases of its reflection and transmission coefficients.

All measurements are subject to uncertainty and ideally the uncertainty in a measurement should be evaluated and reported as part of the measurement result. The evaluation of the uncertainty in microwave S-parameter measurements made using a VNA is a non-trivial task. Published guidance documents [1-3] and software $[4,5]$ to assist users in carrying out this task are available. At the National Physical Laboratory (NPL), when S-parameter measurements are supplied to customers, the measurement uncertainty is evaluated using the Primary Impedance Microwave Measurement System (PIMMS) [6].

The use of PIMMS is quite onerous because it involves making multiple repeated calibrations and measurements. The process can take anywhere from a few hours to several days, depending on the number of devices under test (DUTs), number of ports on each DUT and the number of frequency points. The uncertainty, which includes both systematic and random effects, is calculated during a postprocessing stage involving the measured data and characterisations of the VNA and the calibration standards.

Keysight Technologies has recently introduced a new option for their PNA and PNA-X VNAs [7]: the VNA - Dynamic Uncertainty Option (VNA-DUO), also known as "Option 015" and "S93015A". This tool allows the user to characterise the sources of uncertainty in an S-parameter measurement and displays the S-parameters together with their associated measurement uncertainty on the VNA screen in real time.

This report describes a comparison between VNA-DUO (running on a PNA-X network analyser at NPL) and PIMMS (running on a PNA network analyser at NPL). The purpose of the comparison is to validate VNA-DUO prior to its use at NPL for measurements made during research investigations, etc. The Sparameters of several one- and two-port devices fitted with $3.5 \mathrm{~mm}$ precision coaxial connectors were measured using the two systems and the results were compared in terms of the values of the $S$-parameters and the corresponding measurement uncertainties. The VNA-DUO measurements were made using three different calibration methods: (i) a Thru-Reflect-Line (TRL) calibration made using mechanical standards, (ii) a Short-Open-Load-Reciprocal Thru (SOLR) calibration made using mechanical standards, and (iii) a calibration made using an electronic calibration (ECal) module.

\section{EXPERIMENTAL SETUP}

The DUTs used for the investigation were one-port and two-port devices fitted with precision $3.5 \mathrm{~mm}$ coaxial connectors. According to [8], $3.5 \mathrm{~mm}$ connectors operate mode-free up to at least $33 \mathrm{GHz}$. However, $3.5 \mathrm{~mm}$ calibration kits from most vendors such as Keysight, Maury Microwave etc. are usually only characterised and supported up to $26.5 \mathrm{GHz}$. Therefore, the investigation in this report was performed between $0.1 \mathrm{GHz}$ and $26 \mathrm{GHz}$.

The measurements were made using a four-port Keysight N5247A PNA-X VNA which has a frequency range of $10 \mathrm{MHz}$ to $67 \mathrm{GHz}$ and is fitted with $1.85 \mathrm{~mm}$ coaxial connectors. To allow the DUTs to be connected to the VNA, $1.85 \mathrm{~mm}$ to $3.5 \mathrm{~mm}$ adaptors were used on VNA test ports 1 and 2. A flexible coaxial cable with good amplitude and phase stability was used on VNA test port 2 . Port 1 of the VNA consisted of a $3.5 \mathrm{~mm}$ male test port and port 2 of the VNA consisted of a $3.5 \mathrm{~mm}$ female test port. 
The VNA settings used during the measurements were as follows:

1. Frequency range: $100 \mathrm{MHz}$ to $26 \mathrm{GHz}$

2. Frequency step: $50 \mathrm{MHz}$ below $1 \mathrm{GHz}$ and $0.2 \mathrm{GHz}$ above $1 \mathrm{GHz}$

3. Power: $-5 \mathrm{dBm}$

4. IF Bandwidth: $10 \mathrm{~Hz}$

5. Averaging Factor: 2

The measurements were carried out in a temperature-controlled laboratory at a temperature of $23 \pm 2{ }^{\circ} \mathrm{C}$. A photograph of the measurement set-up is shown in Figure 1. A photograph of a two-port DUT connected to the VNA test ports is shown in Figure 2.

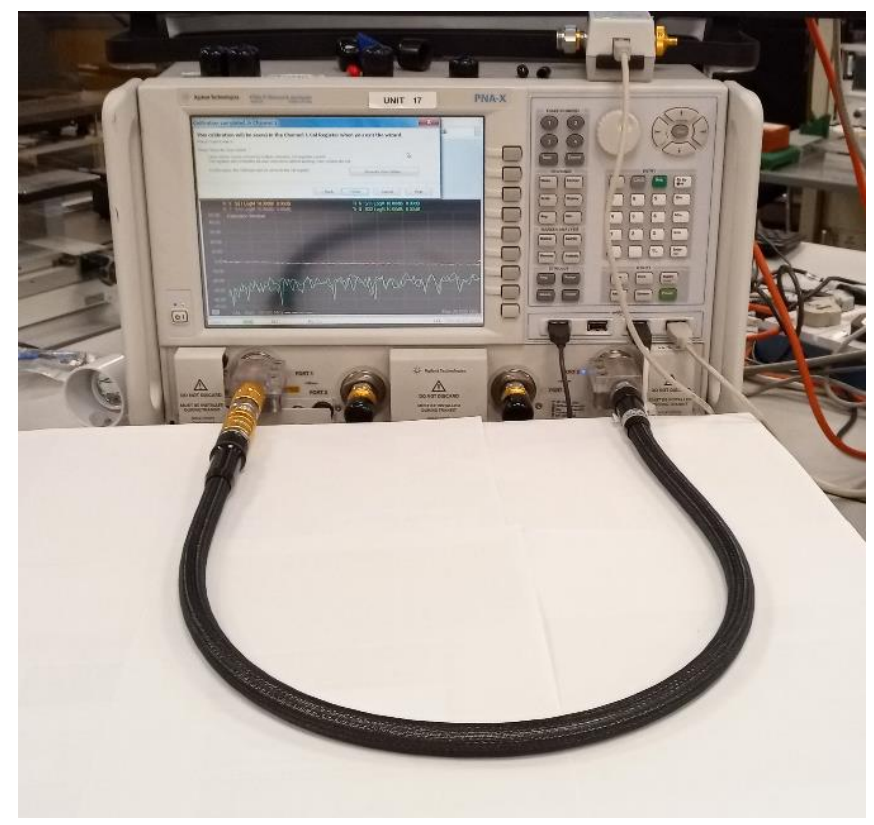

Figure 1: VNA measurement set-up

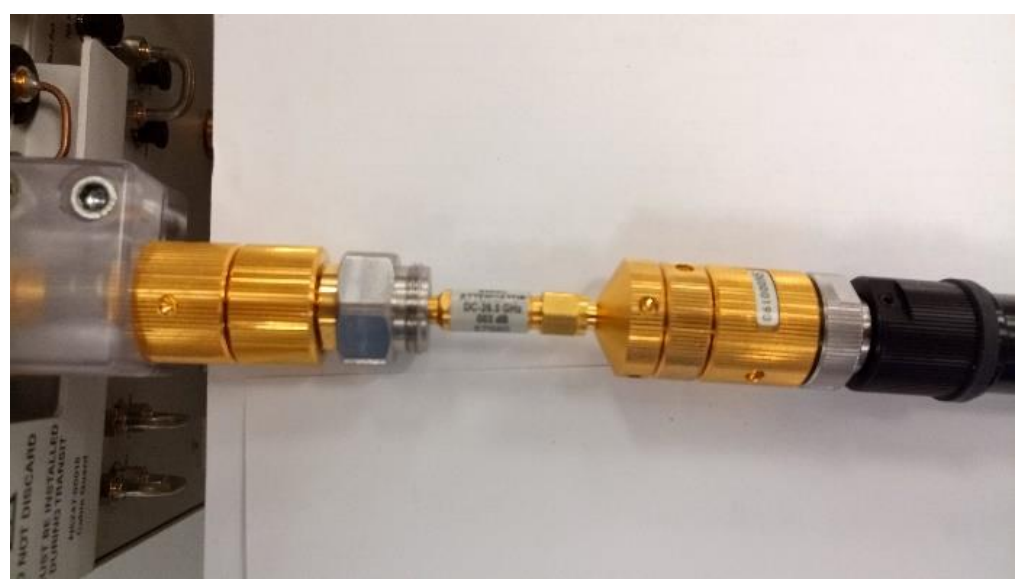

Figure 2: 2-port DUT connected to the VNA test ports 


\subsection{DEVICES UNDER TEST}

The one-port and two-port DUTs measured in this investigation are listed in Table 1 and Table 2 respectively. The DUTs were chosen to provide a wide range of reflection and transmission coefficient values. Typical reflection and transmission performance of the DUTs are plotted in Figure 3 and Figure 4 , respectively.

Table 1: One-port DUTs measured in this investigation

\begin{tabular}{|l|l|l|}
\hline One-port DUTs & Model Number & Description \\
\hline Offset Open-Circuit \#1 & MMC-8048A1 & Open (F) with a 5.25 mm offset line \\
\hline Offset Open-Circuit \#2 & MMC-8048B1 & Open (M) with a 22.75 mm offset line \\
\hline Offset Short-Circuit \#1 & MMC-8046F & Short (F) with a 5 mm offset line \\
\hline Offset Short-Circuit \#2 & MMC-8047F & Short (M) with 40 mm offset line \\
\hline 50 Ohm Load & MMC-8031A5 & 50 $\Omega$ Load (M) \\
\hline Check Standard \#1 & NPL-35F1 & NPL 3.5 mm (F) reflection check standard \\
\hline Check Standard \#2 & NPL-35M1 & NPL 3.5 mm (M) reflection check standard \\
\hline
\end{tabular}

Table 2: Two-port DUTs measured in this investigation

\begin{tabular}{|l|l|l|}
\hline Two-port DUTs & Part Number & Description \\
\hline Thru & N/A & Direct connection of the VNA test ports \\
\hline Beatty Line & HP-85053-60004 & Coaxial Beatty Line \\
\hline $3 \mathrm{~dB}$ Attenuator & Agilent $-8493 \mathrm{C}$ & Attenuator \\
\hline $20 \mathrm{~dB}$ Attenuator & HP-85053-60001 & Attenuator \\
\hline $40 \mathrm{~dB}$ Attenuator & HP-85053-60002 & Attenuator \\
\hline
\end{tabular}

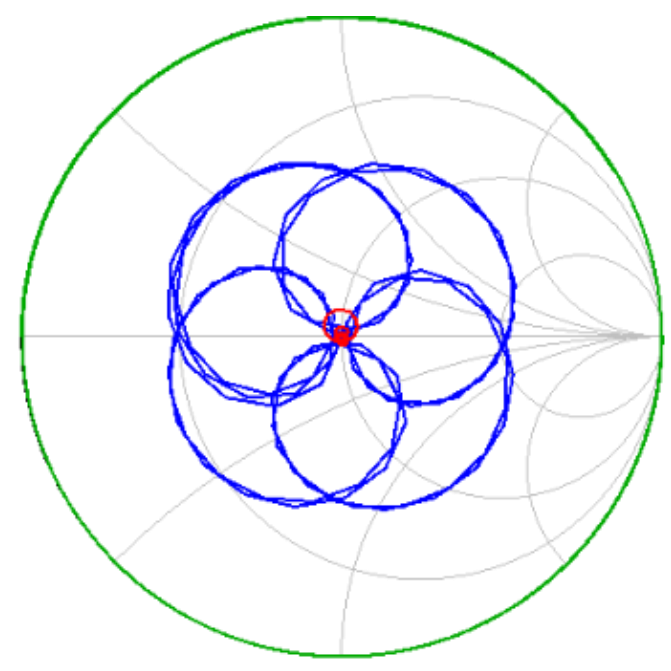

Figure 3: Typical reflection coefficients of one-port and two-port DUTs plotted from $100 \mathrm{MHz}$ to $26 \mathrm{GHz}$ on a Smith chart. The DUTs are: green - offset short-circuit; blue - Beatty line; red - Reflection Check Standard. 


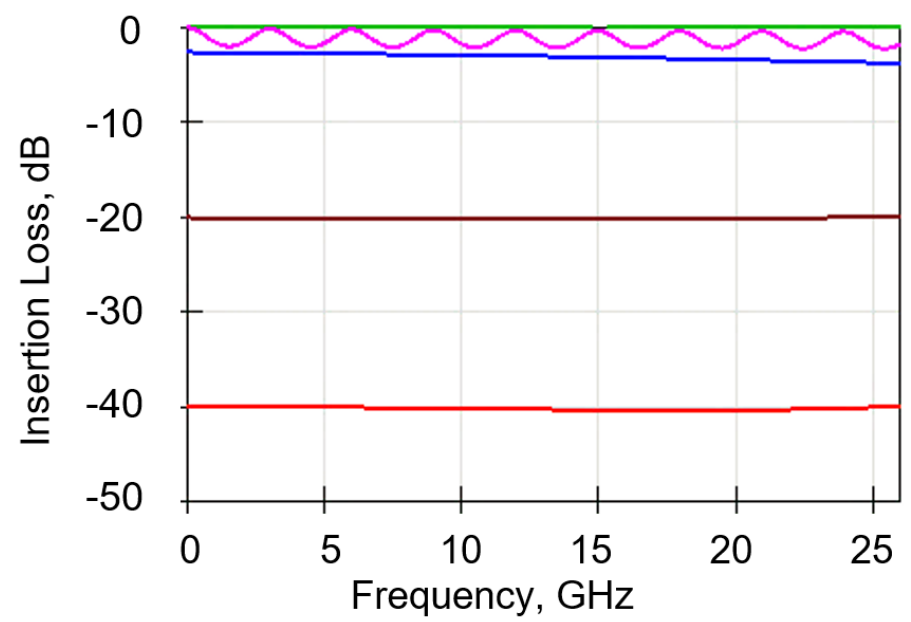

Figure 4: Typical insertion loss of two-port DUTs plotted from $100 \mathrm{MHz}$ to $26 \mathrm{GHz}$. The DUTs are: green - thru; pink - Beatty line; blue - $3 \mathrm{~dB}$ attenuator; brown - $20 \mathrm{~dB}$ attenuator; red - $40 \mathrm{~dB}$ attenuator.

\subsection{CALIBRATION TYPES}

VNA-DUO supports the following calibration types: Line-Reflect-Match (LRM), Thru-Reflect-Line (TRL), Short-Open-Load-Reciprocal-Thru (SOLR), Quick-Short-Open-Load-Thru (QSOLT) and Electronic Calibration (ECal). Of these, TRL, SOLR and ECal were selected for study in this investigation.

The calibration standards used are listed in Table 3. The calibration standards for the SOLR calibration were taken from a $3.5 \mathrm{~mm}$ calibration kit, Model 8050C, from Maury Microwave Corp. ${ }^{1}$ The thru used for the SOLR calibration was a zero length (flush) thru. Three TRL line standards were used to cover the frequency range $100 \mathrm{MHz}$ to $26 \mathrm{GHz}$. Short-circuits were used as the TRL reflect standards.

Table 3: Calibration standards used for SOLR, TRL and ECal calibrations

\begin{tabular}{|l|l|}
\hline Calibration standard & Description \\
\hline Zero-length Thru & Two VNA test ports connected together \\
\hline Open-circuit & Two open-circuits (one male and one female) \\
\hline Short-circuit & Two short-circuits (one male and one female) \\
\hline $50 \Omega$ Loads & Two loads (one male and one female) \\
\hline Line \#1 (frequency range 0.1 to $1.5 \mathrm{GHz}$ ) & $74.93 \mathrm{~mm}$ coaxial air line \\
\hline Line \#2 (frequency range 1.5 to $7.0 \mathrm{GHz}$ ) & $16.17 \mathrm{~mm}$ coaxial air line \\
\hline Line \#3 (frequency range 7.0 to $26 \mathrm{GHz}$ ) & $3.90 \mathrm{~mm}$ coaxial air line \\
\hline $\begin{array}{l}\text { ECal module (frequency range } 300 \mathrm{kHz} \text { to } \\
26.5 \mathrm{GHz})\end{array}$ & Keysight ECal module N4691D-60004 \\
\hline
\end{tabular}

\footnotetext{
${ }^{1}$ This kit has NPL reference number CIS/C/306.

2 This ECal module has serial number11728.
} 
BACKGROUND TO VNA-DUO

VNA-DUO considers three sources of uncertainty in S-parameter measurements: 1) uncertainty due to the calibration standards, 2) uncertainty due to the VNA system noise, and 3) uncertainty due to cable and connector repeatability. The evaluation of the uncertainty due to these sources is treated in depth, in [9]. These sources of uncertainty are discussed below.

(i) Calibration Standards

To calibrate the VNA for S-parameter measurements, calibration standards with known (or partially known) characteristics are used. The uncertainties in the definitions of the calibration standards are propagated to the uncertainty in the calibration coefficients of the VNA and hence to the uncertainty in the S-parameters of the DUT. The characteristics of the calibration standards need to be determined with uncertainties, for use with VNA-DUO.

(ii) System Noise

The two types of VNA system noise are: 1) low-level noise or background (thermal) noise due to the measurement channel equivalent noise figure, and 2) high-level noise or jitter noise due to the local oscillator (LO) source phase noise. Both types of noise need to be characterised for use with VNA-DUO.

(iii) Cable and Connector Repeatability

Cable and connector repeatability are random effects due to imperfections in the cables and connectors used for the VNA measurements. In a 2-port measurement, a cable is usually required on at least one of the VNA ports. High quality phase stable RF cables are generally used. Cable and connector repeatability need to be characterised for use with VNA-DUO.

Other sources of uncertainty such as drift, temperature stability, linearity, etc, are not considered by VNA-DUO but the effect of these can be minimised by:

- Carrying out the measurements in a temperature-controlled environment;

- Making measurements as soon after the calibration as possible to reduce drift effects;

- Operating the VNA in its linear region.

The overall measurement uncertainty for each S-parameter is calculated and displayed by VNA-DUO based on all three of the sources of uncertainty mentioned above. The measurement uncertainty is displayed in real time together with the measurement result.

VNA-DUO can display S-parameters and the corresponding uncertainties in the standard formats such as Cartesian (real and imaginary components), polar (linear magnitude and phase), log magnitude (dB), Smith chart, etc.

\section{CHARACTERISATION OF THE SOURCES OF UNCERTAINTY}

As discussed in Section 4, three sources of measurement uncertainty are considered by VNA-DUO: uncertainty due to the definitions of the calibration standards, uncertainty due to the VNA system noise and uncertainty due to connector and cable repeatability. Each of these sources of uncertainty were fully characterised for the measurement setup used in this investigation as explained in the following subsections. 


\subsection{UNCERTAINTY DUE TO CALIBRATION STANDARDS}

Three methods have been used to define calibration standards with uncertainty for use with VNA-DUO: (i) calibration standard definitions supplied by the manufacturer, (ii) calibration standard definitions based on mechanical dimensions and (iii) calibration standard definitions based on PIMMS measurements. These three methods of defining the calibration standards with uncertainty are described in more detail below and are compared in Section 6. The contribution of individual calibration standards to the overall S-parameter measurement uncertainty is investigated in Section 9.

\subsubsection{Calibration standard definitions supplied by the manufacturer}

VNA-DUO includes definitions, with uncertainties, of several Keysight calibration kits (including the $3.5 \mathrm{~mm}$ calibration kit $85052 \mathrm{C}$ used in this investigation). However, as described in Section 6, it was found that using these manufacturer-supplied definitions resulted in S-parameter uncertainties for the DUTs that were considered to be too large.

\subsubsection{Calibration standard definitions based on mechanical dimensions}

Calibration standards can also be defined by their respective physical dimensions. The uncertainty in these standards are due to manufacturing tolerances. The coaxial standards are defined by the outer conductor and inner conductor diameters and length. The nominal tolerances for these dimensions are $\pm 3 \mu \mathrm{m}$.

\subsubsection{Calibration standard definitions based on PIMMS measurements}

All the calibration standards (open, load, short, thru, and three air lines) were characterised (i.e. measured) over the full frequency range using PIMMS. For each calibration standard, the PIMMS S-parameter data with uncertainties was converted and saved in .dsd format (see the Appendix for format details), for importing into VNA-DUO.

\subsubsection{ECal Module}

For the ECal module, only the definitions and uncertainties supplied by the manufacturer were used.

\subsection{UNCERTAINTY DUE TO VNA SYSTEM NOISE}

The VNA high-level noise (trace noise) and low-level noise (noise floor) are measured and the noise characterisation is used in the uncertainty analysis over the measured frequency range. Both ports of the VNA were measured independently with the IF bandwidth (IFBW) set to $10 \mathrm{~Hz}$. VNA-DUO allows the noise characterisation to be performed at an IFBW within a factor of ten of the IFBW used during the measurement. The noise floor was characterised with $50 \Omega$ loads connected to both ports and the trace noise was characterised with short-circuits connected to both ports. The load and short-circuit standards from the $85052 \mathrm{C}$ calibration kit were used for this purpose.

\subsection{UNCERTAINTY DUE TO CONNECTOR AND CABLE REPEATABILITY}

Port one of the VNA was fitted with a ruggedized RF connector and a flexible RF cable was used on port two of the VNA. The cable flexing and re-connection uncertainty on both ports were characterised by performing a series of repeat measurements. The ruggedized RF connector on port one was assigned as a "zero-length" test cable and the flexible RF cable on port two was assigned as a $3.5 \mathrm{~mm}$ RF cable. Each of the two ports were characterised independently by measuring 20 repeated load re-connections and 20 repeated short-circuit re-connections. Each time a re-connection was made the terminating device was also arbitrarily rotated to take account of all aspects of the connector repeatability. During repeatability characterisation, the flexible RF cable connected to port two was also moved to different positions as shown in Figure 5 to take account of uncertainties due to cable flexing. The repeatability 
characterisation was done across the measurement frequency range with the VNA configured as for the DUT measurements.

It should be noted that VNA-DUO estimates the contribution of connector repeatability to the Sparameter measurement uncertainty using repeat measurements on a "Load" and a "Short", which are usually high precision components, taken from a calibration kit. The connector repeatability of the actual DUT, which may be different from these calibration kit components, is not measured directly in VNA-DUO. On the other hand, in PIMMS, repeat measurements are made on the actual DUT to estimate the contribution of connector repeatability to the overall uncertainty. This difference in procedure (between PIMMS and VNA-DUO) may lead to different values of uncertainty for the repeatability assessments. In particular, VNA-DUO may under estimate the uncertainty, for some DUTs (e.g. where the connetors are no longer in pristine condition).

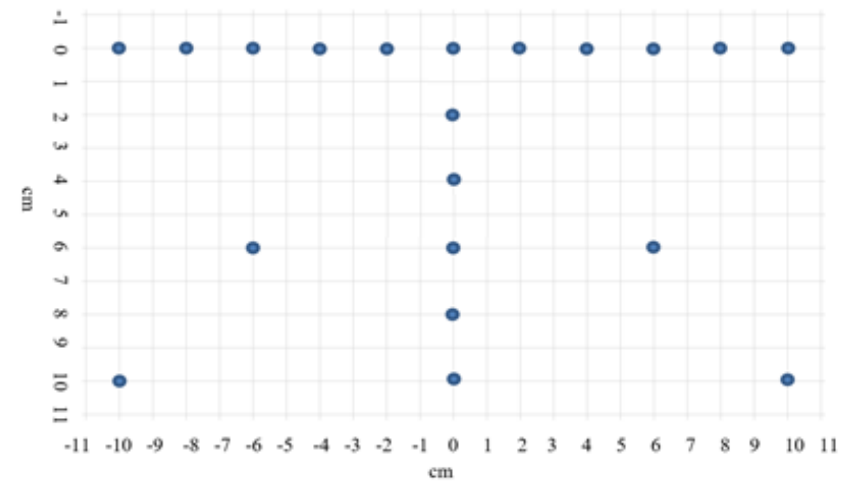

Figure 5: 20 locations of the port-two cable used during cable repeatability characterisation.

\section{COMPARISON OF THREE DIFFERENT METHODS FOR CHARACTERISING THE CALIBRATION STANDARDS}

To compare the different methods of defining calibration standards in VNA-DUO, the calibration standards, listed in Table 3, were defined using three methods; a) manufacturer (Keysight) pre-defined definitions, b) definitions based on mechanical dimensions, and c) definitions based on PIMMS measurements.

Four sets of S-parameter measurements were made of a Beatty line. Three of the sets of measurements were made with VNA-DUO using SOLR calibrations with the calibration standards defined using the three different methods. The fourth set of measurements was made using PIMMS. The measured $S$-parameters $\left(S_{22}\right.$ and $\left.S_{21}\right)$ of the Beatty line are plotted in Figure 6 and Figure 7 . The magnitude and phase of reflection and transmission coefficients are shown for the four measurements, as well as the measurement uncertainties, and, the differences between the VNA-DUO measurements and the PIMMS measurement.

The results show that the measurements based on the Keysight definition of the calibration standards (Keysight) has the largest uncertainties and the measurements based on the mechanical definitions of the standards (mech) show the smallest uncertainties. The measurements based on the PIMMS definition of the calibration standards (NPL PIMMS) show similar uncertainties to those for the direct measurement of the DUT using PIMMS (Ref PIMMS). The magnitude and phase differences for both transmission and reflection coefficients are much smaller than the uncertainty values which indicate 
good agreement between the measurements. A similar investigation was carried out for TRL calibration and similar results were obtained although the results are not given here.

It is clear from the results that calibration kit definitions based on mechanical dimensions and on the generic manufacturer's data provided by Keysight are giving values of uncertainty which are either much smaller or much larger than the uncertainties from the PIMMS measurements. Therefore, for further investigation of VNA-DUO reported here, PIMMS measurement-based calibration standard definitions will be used. These definitions are fully traceable to primary national measurement standards.
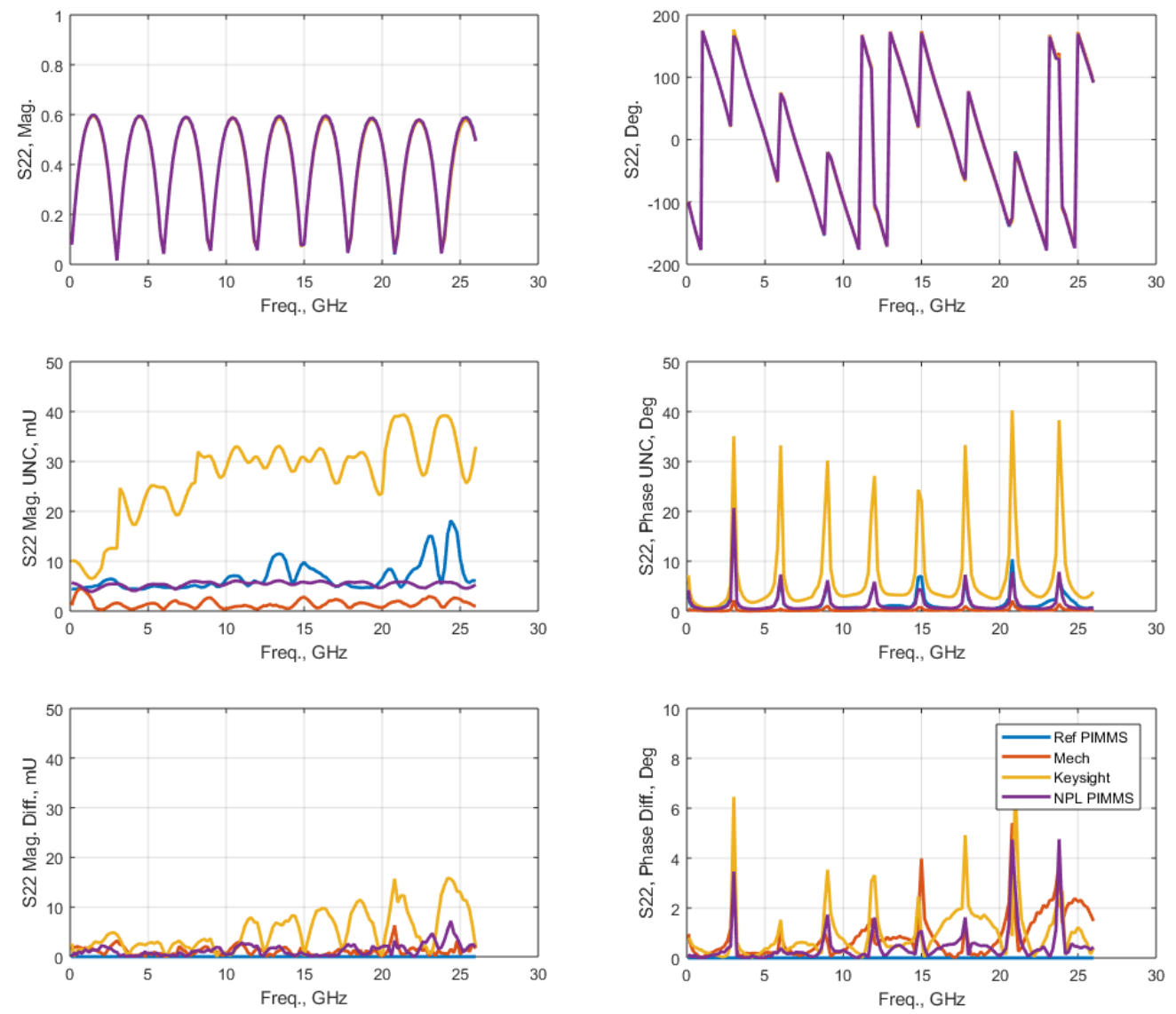

Figure 6: The magnitude and phase of $S_{22}$, the associated measurement uncertainty (UNC), and, the difference from PIMMS measurements (Diff) of a Beatty Line measured using different calibration standard definitions. 

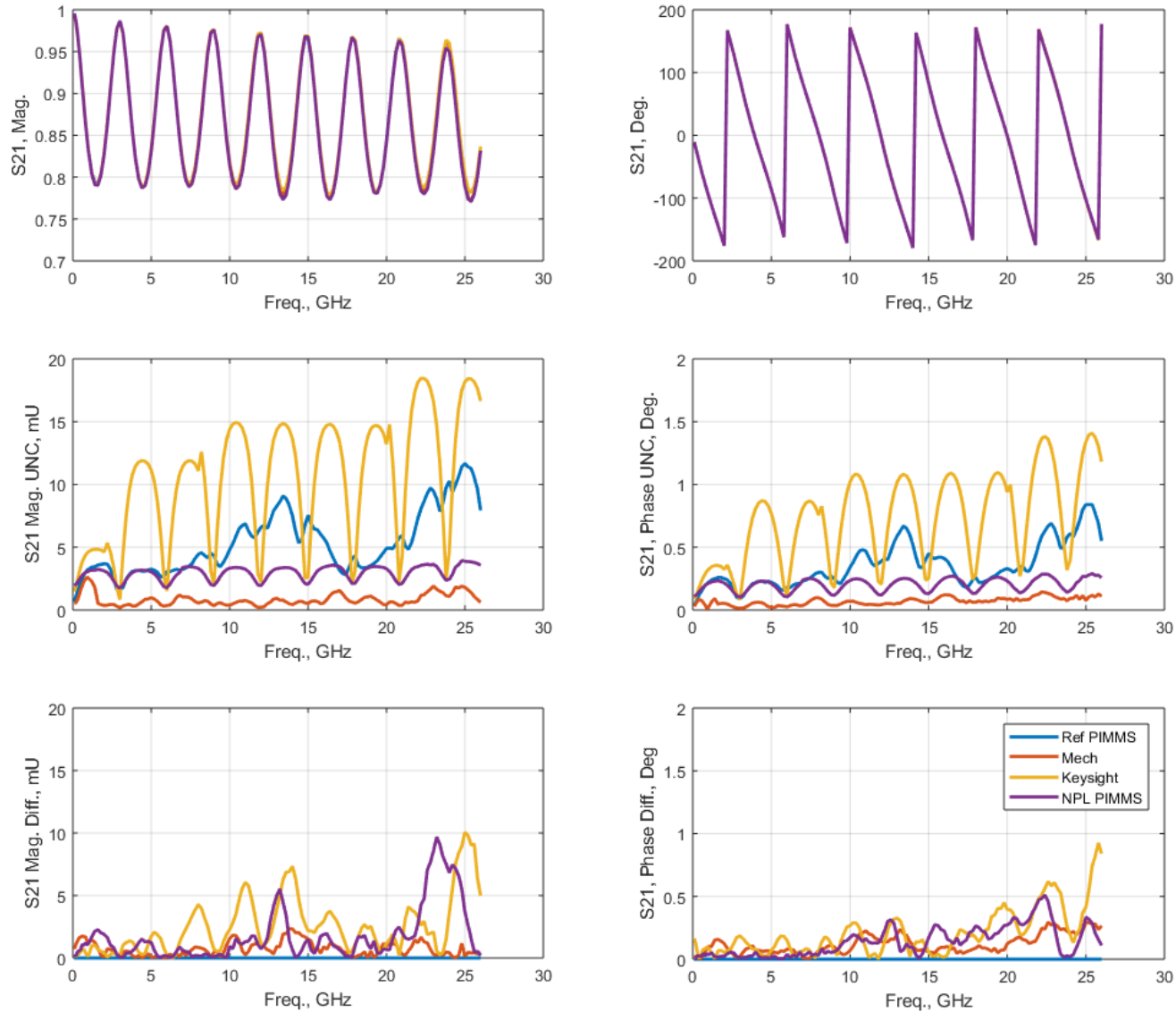

Figure 7: The magnitude and phase of $S_{21}$, the associated measurement uncertainty (UNC), and the difference from PIMMS measurements (Diff) of a Beatty Line measured using different calibration standard definitions.

\section{VNA-DUO MEASUREMENT RESULTS}

VNA-DUO was evaluated by using it to measure the S-parameters of several 1-port and 2-port DUTs listed in Table 1 and Table 2 using the three calibration methods listed in Section 3.2 Error! Reference source not found.namely TRL, SOLR and ECal.

As discussed in Section 6, the mechanical standards for the TRL and SOLR calibrations were characterised, with uncertainties, based on PIMMS measurements of the S-parameters of the standards. For the ECal module, the manufacturer's characterisation, with uncertainties, was used. The experimental setup was as described in Section 2. The VNA-DUO measurements were compared with PIMMS measurements of the S-parameters of the same DUTs. The expanded uncertainty with level of confidence of $95 \%$ in all measurements has been compared in this report.

The VNA was calibrated with VNA-DUO using SOLR, TRL and ECal calibration methods. For each calibration method, the calibration coefficients (error terms) were saved to the VNA firmware. Then, for each DUT, the following procedure was followed:

(i) the DUT was connected to the VNA, its raw S-parameters were measured and the VNA was put into hold;

(ii) each of the three calibrations (TRL, SOLR and ECal) were recalled in turn and the calibrated S-parameters with respect to each of the calibrations were saved together with the evaluated 
uncertainties.

Female DUT ports were connected directly to Port 1 of the VNA whilst male DUT ports were connected to Port 2 of the VNA by means of a phase stable cable. The measurements were carried out with minimum movement in the cable.

Each measurement method was compared against PIMMS (taken as a reference) by evaluating, for each DUT S-parameter, the difference in magnitude and the difference in phase for the S-parameter between the measurement method and PIMMS as follows

$$
\begin{aligned}
& (\Delta|S|)_{i}=|S|_{i}-|S|_{\text {PIMMS }} \\
& (\Delta \phi)_{i}=\phi_{i}-\phi_{\text {PIMMS }}
\end{aligned}
$$

where $|S|_{i}$ is the magnitude of the S-parameter for method $i$ measured using VNA-DUO and $|S|_{\text {PIMMS }}$ is the magnitude measured using PIMMS and, similarly $\phi_{i}$ is the phase of the S-parameter for method $i$ measured using VNA-DUO and $\phi_{P I M M S}$ is the phase measured using PIMMS.

The S-parameter measurements of the DUTs in this investigation have already been summarised in [10] including differences in S-parameters between the different calibration methods. So, in this report, more emphasis is given to the measurement uncertainty evaluated for the different calibration types.

The magnitude and phase of the measured reflection and transmission coefficients, the corresponding measurement uncertainties, and the differences between the VNA-DUO and PIMMS measurements are plotted in Figure 8Figure 8 to Figure 14 for the one-port DUTs and in Figure 15 to Figure 24 for the two-port DUTs. For S-parameters of small magnitude, corresponding to points close to the origin in the complex plane, the phase is not well defined. A small perturbation in the value of the S-parameter can result in a very large change in the value of its phase. For this reason, phase is not considered for Sparameters of small magnitude e.g. for the reflection coefficient of a well-matched device such as a $50 \Omega$ load or a precision attenuator.

In order to compare S-parameter measurements made using VNA-DUO with those made using PIMMS, the normalised error ratio (NER) was computed for the magnitude and phase of each DUT S-parameter, at each frequency, as follows

$$
N E R_{i}=\frac{\left|S_{i}-S_{\text {PIMMS }}\right|}{\sqrt{\left(U n c_{i}{ }^{2}+U n c_{\text {PIMMS }}{ }^{2}\right)}}
$$

where $S_{i}$ is the magnitude or phase of the $S$-parameter measured using calibration method $i$ (i.e. SOLR, TRL or ECAL) in VNA-DUO, SPIMMs is the magnitude or phase of the S-parameter measured using PIMMS, $\mathrm{Unc}_{i}$ is the measurement uncertainty for method $i$ in VNA-DUO and Unc measurement uncertainty for PIMMS. Both $\mathrm{Unc}_{\mathrm{i}}$ and Unc $\mathrm{UIMMS}_{\mathrm{P}}$ are expanded uncertainties with a level of confidence of $95 \%$. NER is commonly used to evaluate the consistency of two measurements with an NER value less than one being considered to indicate that the measurements agree within their uncertainties.

\subsection{ONE-PORT DEVICES}

Seven one-port devices providing a range of reflection coefficient values were measured (see Table 1). The measured results for the one-port devices are discussed in the following sub-sections. 


\subsubsection{Offset Open-Circuits}

Two open-circuits (one male and one female) were measured during this investigation; open-circuit \#1 was a standard open-circuit with a $5.25 \mathrm{~mm}$ offset whilst open-circuit \#2 had an additional adaptor to provide extra phase length, totalling an offset of $22.75 \mathrm{~mm}$. The magnitude and phase of $\mathrm{S}_{11}$, the difference relative to the PIMMS measurements, measurement uncertainties and NER of the magnitude and phase for the two open-circuit DUTs are plotted in Figure 8 and Figure 9 for all the calibration methods.

The results show that the magnitudes measured using each calibration are all within $15 \mathrm{mU}$ of the reference PIMMS measurement, for most of the frequency range, and the phases are all within $1.2^{\circ}$ of the reference PIMMS measurement. The measurement uncertainties are less than $20 \mathrm{mU}$ for magnitude and less than $1.5^{\circ}$ for phase. The measurement difference in $S_{11}$ magnitude from the reference PIMMS measurement is smaller than the measurement uncertainty for both TRL and SOLR calibrations. The phase of the second open-circuit shows measurement differences, relative to PIMMS, of larger than the uncertainty. The NER values for the $S_{11}$ phase measurements for the second Short are also greater than one.

The measurement results from the ECal calibration are also plotted. The NER values show that SOLR and TRL measurements agree well with PIMMS, since the NER values are less than one. However, the ECal measurements show NER values greater than one, at some frequencies, mainly due to large differences in the measurements, relative to PIMMS. 

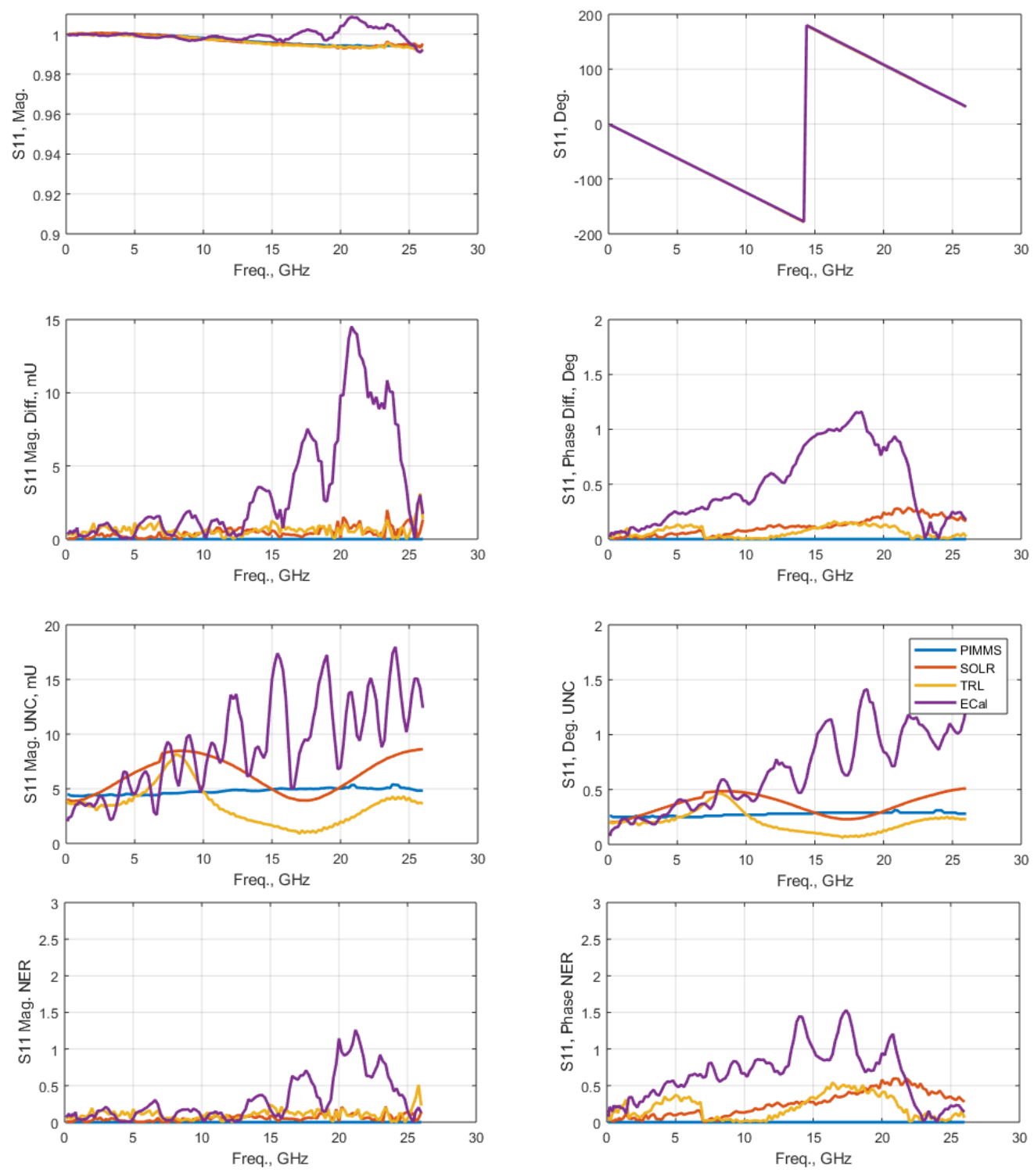

Figure 8: Magnitude and phase of $S_{11}$, difference from PIMMS (Diff), measurement uncertainty (UNC) and NER for $S_{11}$ of offset open-circuit \#1. 

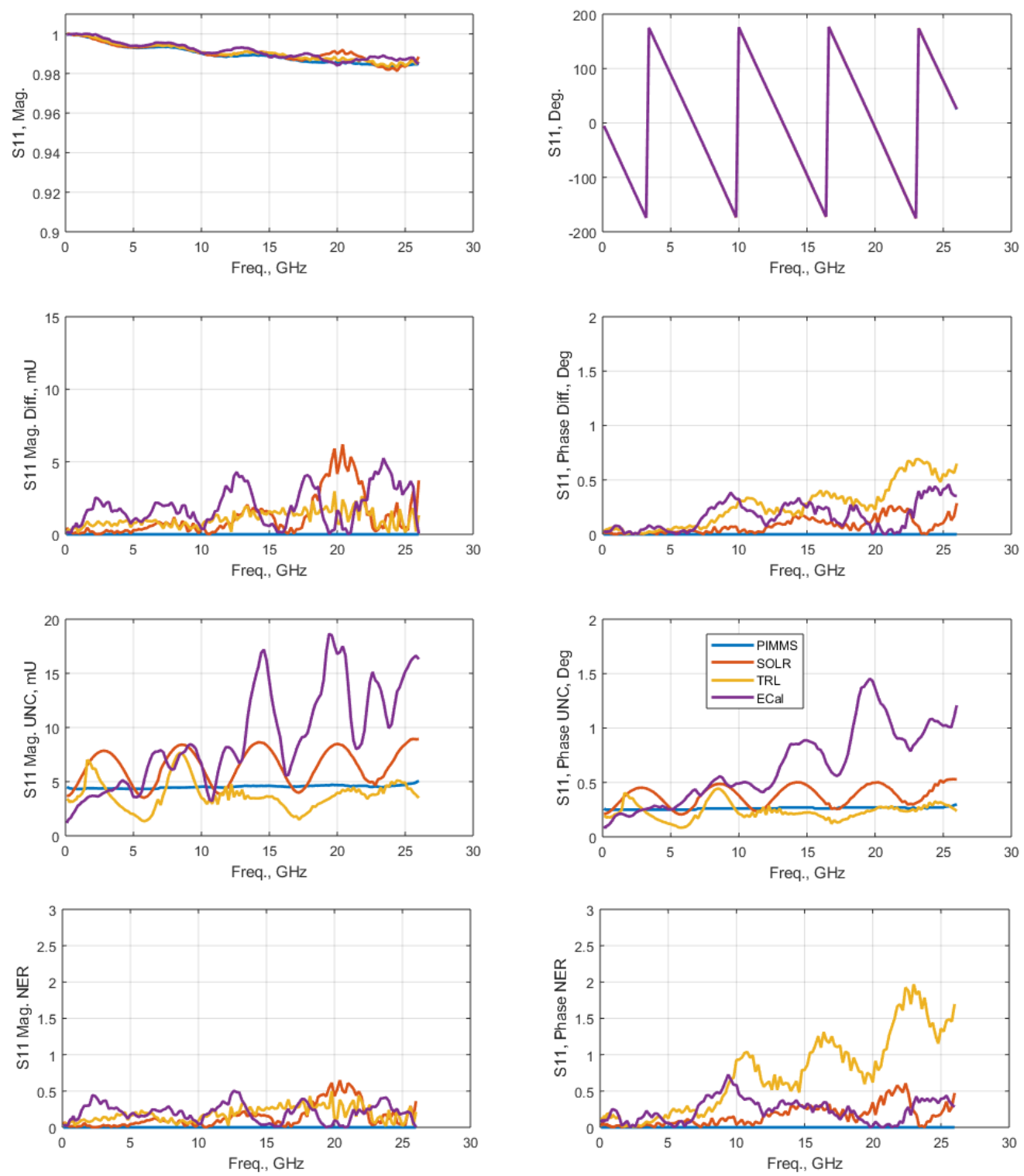

Figure 9: Magnitude and phase of $\mathrm{S}_{11}$, difference from PIMMS (Diff), measurement uncertainty (UNC) and NER for $S_{11}$ of offset open-circuit \#2.

\subsubsection{Offset Short-Circuits}

Two short-circuits (one male and one female) were measured during this investigation; short-circuit \#1 was a $5 \mathrm{~mm}$ offset short-circuit whilst short-circuit \#2 had an additional adaptor to provide extra phase length, totalling an offset of $40 \mathrm{~mm}$. The magnitude and phase of $S_{11}$, the differences relative to the PIMMS measurements, the measurement uncertainties and NER of the magnitude and phase of $S_{11}$ for the two short-circuits are plotted in Figure 10 and Figure 11 for all the calibration methods.

The uncertainties for the SOLR calibration are mostly larger than those for the PIMMS calibration but the TRL measurement uncertainties at low frequencies are smaller than the PIMMS uncertainties. The $S_{11}$ magnitude and phase measurement differences are smaller than the uncertainty for all SOLR results. TRL uncertainties are much smaller than the PIMMS uncertainties at troughs and also the phase of the second short-circuit shows measurement differences, relative to PIMMS, that are larger than the 
uncertainty. The NER values for the $S_{11}$ phase measurements for the second Short are also greater than one.

The results show that the measurement uncertainties for the ECal calibrated results are larger than for the other calibration types. The ECal results also show odd behaviour at around $22 \mathrm{GHz}$. The ECal results also show differences in the measurements with respect to the reference PIMMS measurements that are larger than the uncertainties, resulting in NER values that are greater than one at those frequencies.
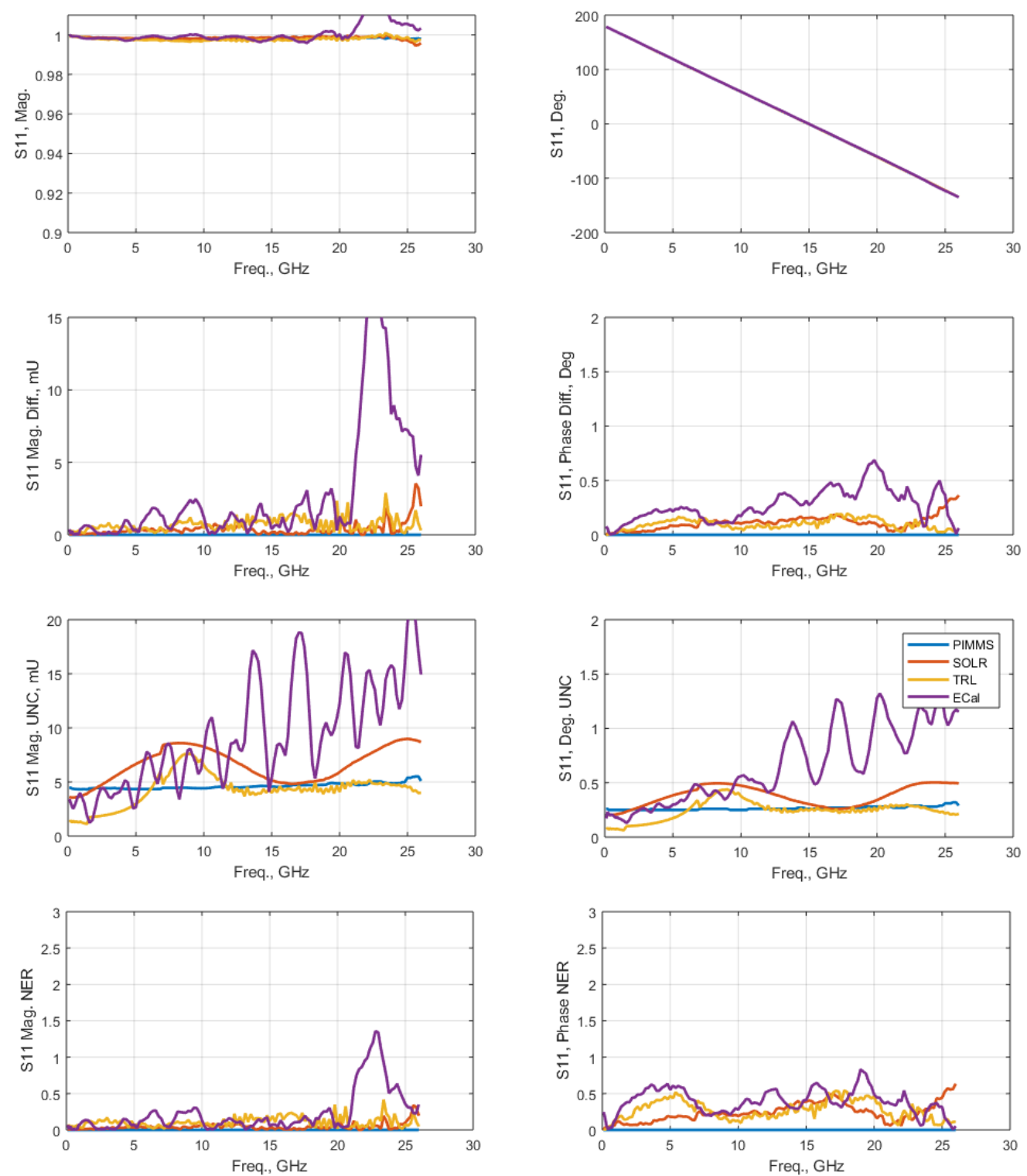

Figure 10: Magnitude and phase of $S_{11}$, difference from PIMMS (Diff), measurement uncertainty (UNC) and NER for $S_{11}$ of offset short-circuit \#1. 

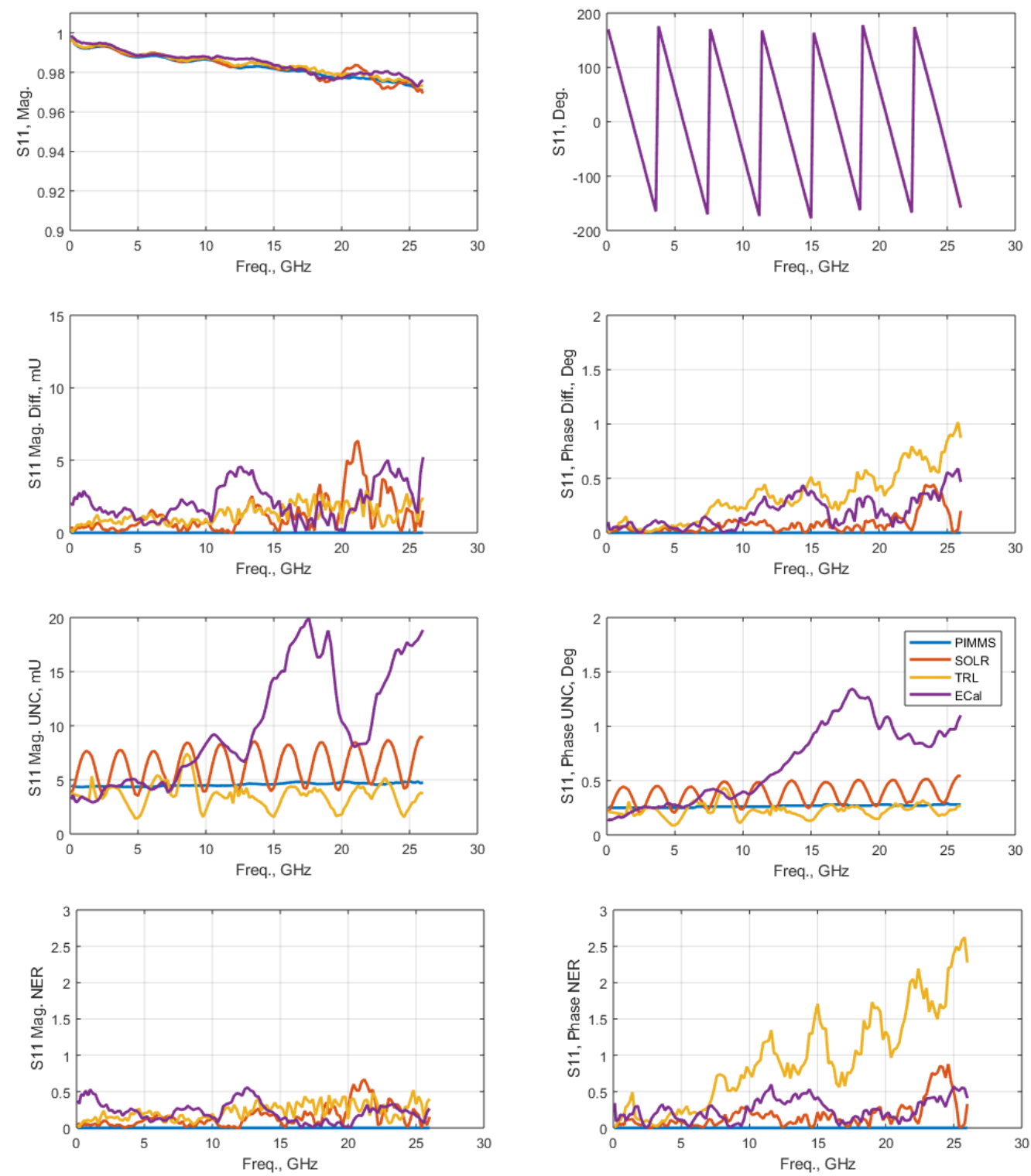

Figure 11: Magnitude and phase of $S_{11}$, difference from PIMMS (Diff), measurement uncertainty (UNC) and NER for $S_{11}$ of offset short-circuit \#2.

\subsection{3 $50 \mathrm{Ohm}$ Load}

A $50 \Omega$ load (male) was measured during the investigation. The magnitude of $S_{11}$, the differences relative to the PIMMS measurements, the measurement uncertainties and NER are plotted in Figure 12 for all the calibration methods. Note that only magnitude results for the load are shown as reflection coefficient phase for a low reflect DUT can vary widely, due to the measurement error encompassing the centre of the Smith chart.

For each of the calibration methods, the differences in magnitude from the PIMMS measurements are less than $4 \mathrm{mU}$ for most of the frequency range. The TRL measurement uncertainty shows a peak at the lowest frequency which is due to a limitation in the line calibration standard. The measurement uncertainty in the magnitude of $S_{11}$ is less than $5 \mathrm{mU}$ for all the calibration methods. Of the different measurement methods, the PIMMS measurement shows the highest measurement uncertainty. The 
measurement uncertainty in VNA-DUO using SOLR and TRL were expected to be greater than the PIMMS measurements as the calibration standards used in these measurements are based on the PIMMS measurements. The SOLR uncertainties are similar to the PIMMS uncertainties, but the TRL uncertainties can be less than half of the PIMMS uncertainties. This shows that VNA-DUO can produce (unrealistically) small uncertainties for low reflect devices compared to PIMMS. The differences from the reference PIMMS measurements are smaller than the measurement uncertainty for all calibrations.
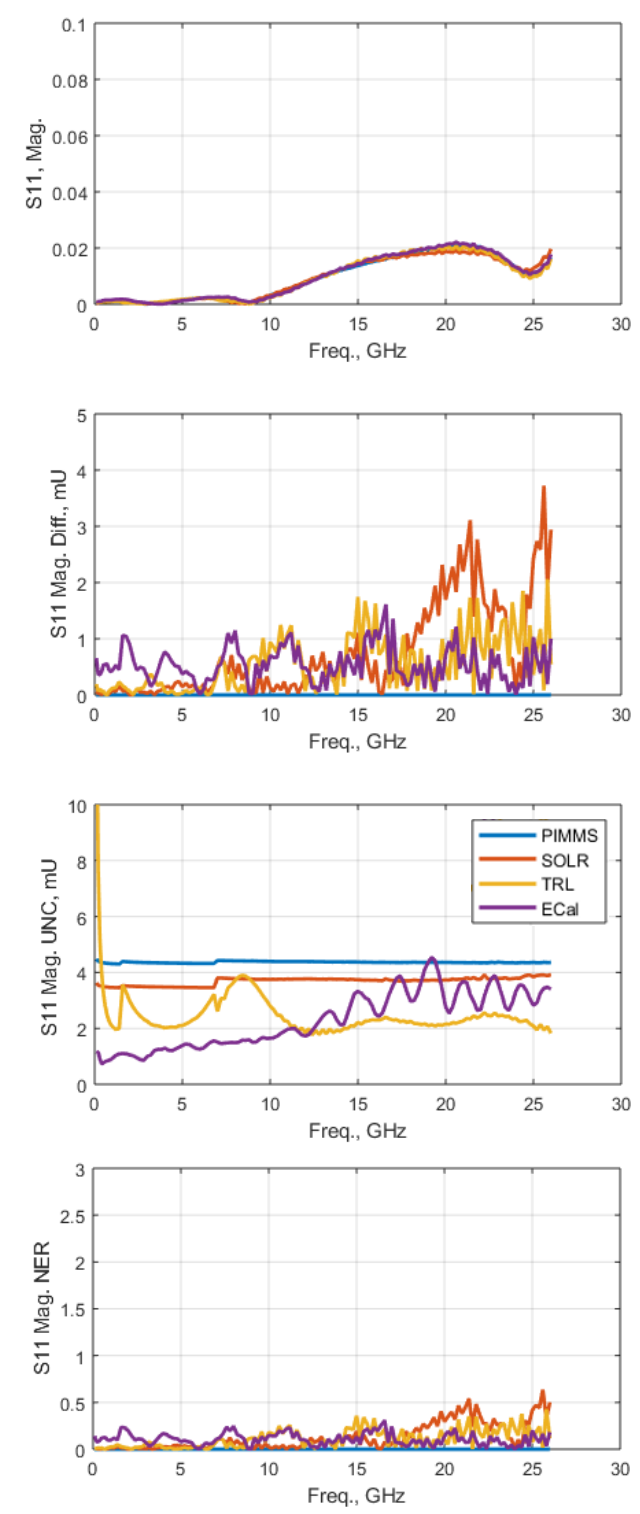

Figure 12: Magnitude of $S_{11}$, difference from PIMMS (Diff), measurement uncertainty (UNC) and NER for the $50 \Omega$ load.

\subsubsection{Reflection Check Standards}

Two one-port reflection check standards were measured during the investigation (one male and one female). These impedance standards, which are periodically characterised in terms of their reflection coefficients, are used at NPL to verify VNA reflection measurements. The magnitude and phase of $S_{11}$, the differences from the PIMMS measurement, the measurement uncertainties and NER values are plotted in Figure 13 and Figure 14 for all the calibration methods. 
The differences between the VNA-DUO measurements and the PIMMS measurements are less than $10 \mathrm{mU}$ for magnitude and less than $7^{\circ}$ for phase. The ECal measurements show larger differences from the PIMMS measurements for the first check standard (35F1).

As for the $50 \Omega$ Load results, the check standard results also show the largest measurement uncertainties for PIMMS, followed by SOLR and then TRL. The differences from the reference PIMMS measurements are smaller than the measurement uncertainty for both TRL and SOLR calibrations.

The Measurement uncertainties for the ECal measurements are small at low frequencies but increase with frequency. The ECal NER values for check standard \#1 are greater than one, at some frequencies.
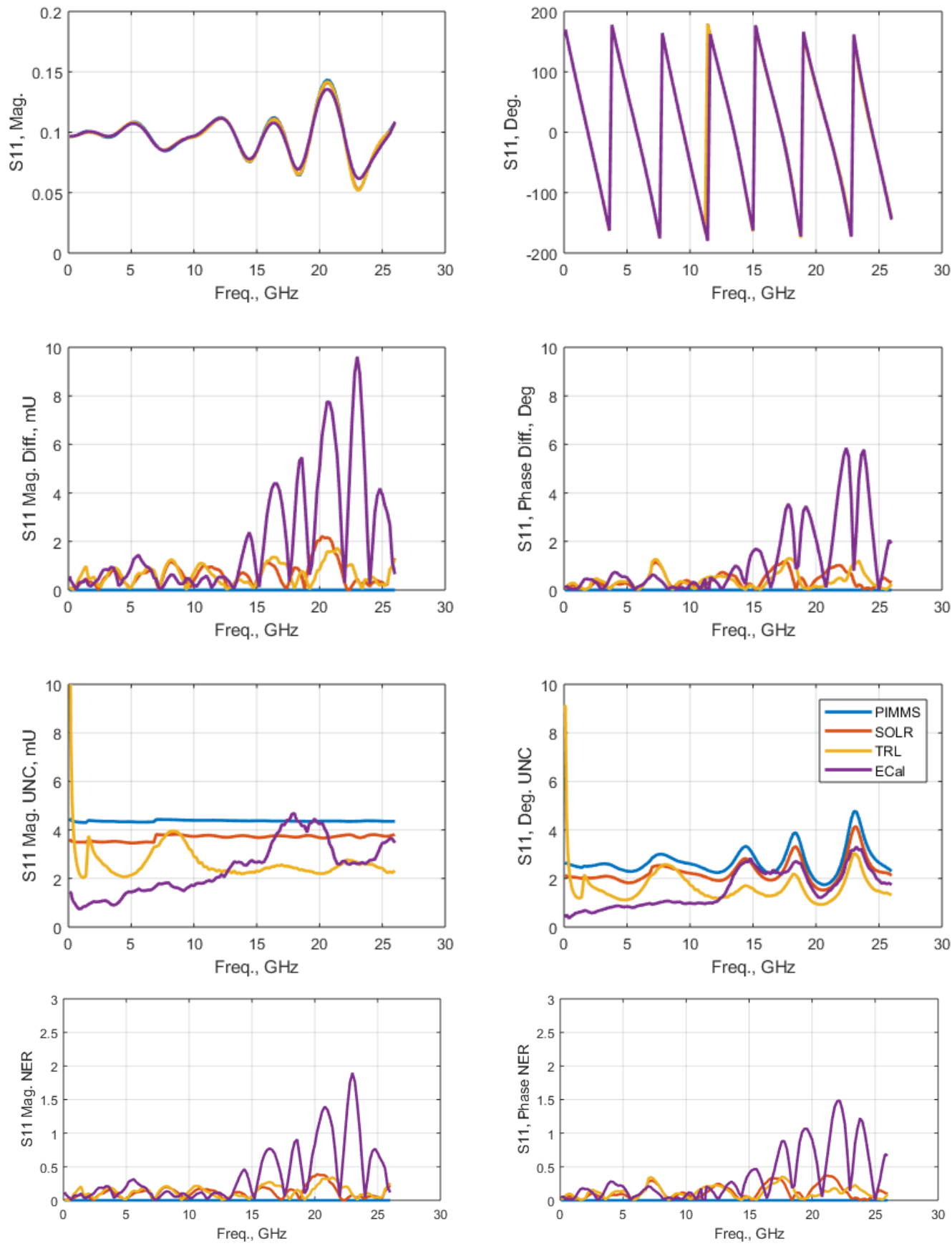

Figure 13: Magnitude and phase of $S_{11}$, difference from PIMMS (Diff), measurement uncertainty and NER for reflection check standard \#1. 

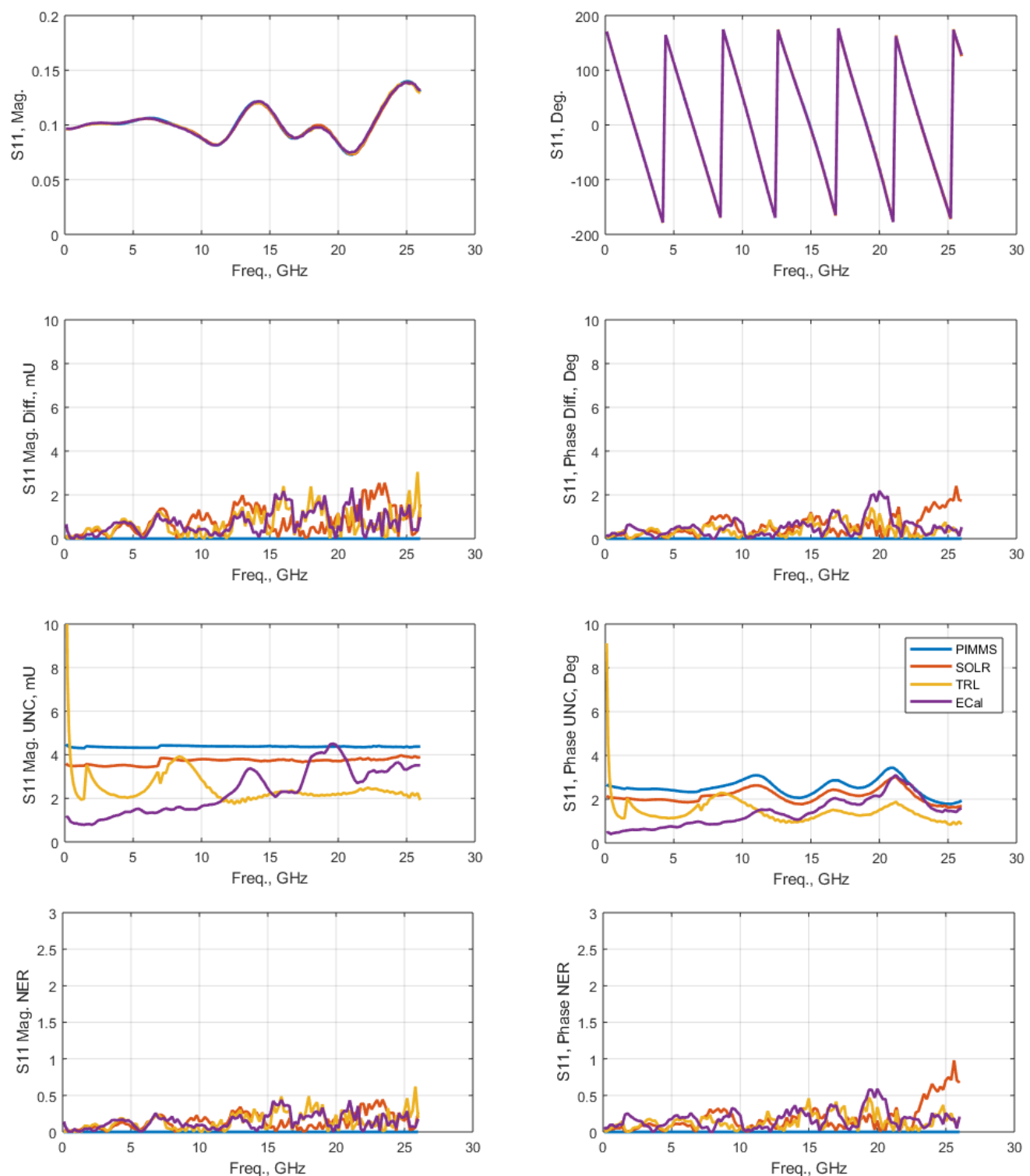

Figure 14: Magnitude and phase of $S_{11}$, difference from PIMMS (Diff), measurement uncertainty (UNC) and NER for reflection check standard \#2.

\subsubsection{Summary of One-Port Device Measurements}

The measurement results for the one-port devices can be summarised by averaging the measurement uncertainties over the measured frequency range. For all devices and all calibration methods, the averaged measurement uncertainties for the $S_{11}$ magnitude component are listed in Table 4; the averaged measurement uncertainties for the $S_{11}$ phase are listed in Table 5.

Two conclusions can be drawn from the measured results of one-port devices using PIMMS and VNA-DUO as follows.

1) For high reflect devices, such as the Opens and Shorts, the averaged VNA-DUO SOLR measurement uncertainties in magnitude are around $1.8 \mathrm{mU}$ larger than those for the PIMMS measuremens. The averaged VNA-DUO TRL measurement uncertainties are smaller than those for the PIMMS measurements (by between $0.5 \mathrm{mU}$ and $1.3 \mathrm{mU}$ ). The averaged measurement uncertainties in magnitude of VNA-DUO ECal are almost twice as large as the PIMMS measurement uncertainties. Similar characteristics are observed for 
the measurement uncertainties in phase.

2) For low reflect devices such as the Load and Check Standards, the averaged VNA-DUO SOLR measurement uncertainties in magnitude are around $0.7 \mathrm{mU}$ lower than those of PIMMS, and the averaged VNA-DUO TRL measurement uncertainties are around $1.8 \mathrm{mU}$ lower than those of PIMMS. The averaged measurement uncertainties in magnitude of VNA-DUO ECal are the lowest of all the methods. Similar characteristics are observed for the measurement uncertainties in phase.

Of the VNA-DUO measurements of the one-port devices, the SOLR method shows measurement uncertainties closest to those of the PIMMS measurements.

Table 4: Measurement uncertainty in $S_{11}$ magnitude $(\mathrm{mU})$ averaged over frequency for each one-port device and calibration method.

\begin{tabular}{|l|r|r|r|r|}
\hline DUT & PIMMS & \multicolumn{1}{l|}{ SOLR } & \multicolumn{1}{l|}{ TRL } & ECal \\
\hline Open \#1 & 4.77 & 6.19 & 3.46 & 8.81 \\
\hline Open \#2 & 4.51 & 6.35 & 3.77 & 8.77 \\
\hline Short \#1 & 4.61 & 6.48 & 4.18 & 9.02 \\
\hline Short \#2 & 4.55 & 6.30 & 3.58 & 9.61 \\
\hline Load & 4.36 & 3.69 & 2.57 & 2.26 \\
\hline 35F1 & 4.37 & 3.68 & 2.75 & 2.36 \\
\hline 35M1 & 4.37 & 3.70 & 2.57 & 2.29 \\
\hline
\end{tabular}

Table 5: Measurement uncertainty in $S_{11}$ phase $\left(^{\circ}\right)$ averaged over frequency for each one-port device and calibration method.

\begin{tabular}{|l|c|c|c|c|}
\hline DUT & PIMMS & SOLR & TRL & ECal \\
\hline Open \#1 & 0.27 & 0.36 & 0.20 & 0.65 \\
\hline Open \#2 & 0.26 & 0.38 & 0.23 & 0.64 \\
\hline Short \#1 & 0.26 & 0.37 & 0.24 & 0.64 \\
\hline Short \#2 & 0.26 & 0.38 & 0.22 & 0.64 \\
\hline Load & - & - & - & - \\
\hline 35F1 & 2.69 & 2.27 & 1.70 & 1.52 \\
\hline 35M1 & 2.52 & 2.14 & 1.50 & 1.36 \\
\hline
\end{tabular}

\subsection{TWO-PORT DEVICES}

Five two-port devices providing a range of reflection and transmission coefficient values were measured (see Table 2). Only the transmission coefficients from port 1 to port $2\left(S_{21}\right)$ are plotted for the two-port DUTs as $S_{12}$ results are expected to be very similar to $S_{21}$. Similarly, only reflection coefficients from port $1\left(\mathrm{~S}_{11}\right)$ are plotted in this report. The measured results for the two-port devices are presented in the following sub-sections.

\subsubsection{Thru}

The Thru measurements were carried out by connecting VNA measurement port 1 directly to VNA measurement port 2 . The Thru measurements were made immediately after the calibrations. 
The magnitude and phase of the reflection coefficient $\left(S_{11}\right)$ and transmission coefficient $\left(S_{21}\right)$ for the Thru, the difference relative to the PIMMS measurements, the measurement uncertainty and NER are plotted in Figure 15 and Figure 16, respectively, for all the calibration methods. The phase of $S_{11}$ for the Thru is not plotted since the Thru is well matched i.e. the magnitude of its reflection coefficient is small.

The results using the ECal unit are significantly different to those obtained from the other calibrations. The results from the SOLR and TRL calibrations show good agreement with the PIMMS results. The SOLR calibrated results show slightly larger uncertainty than the PIMMS results and the TRL calibrated results have slightly lower uncertainties than the PIMMS results. The results of the Thru measurements show that the measurement uncertainties for the TRL calibration are closest to the PIMMS uncertainties and the difference in measurements relative to PIMMS is also lowest for the TRL calibration. The SOLR calibration has uncertainties that are slightly larger than PIMMS, which is to be expected. The ECal NER values for the Thru are greater than one for the magnitude of $S_{21}$ between $20 \mathrm{GHz}$ and 25 $\mathrm{GHz}$.
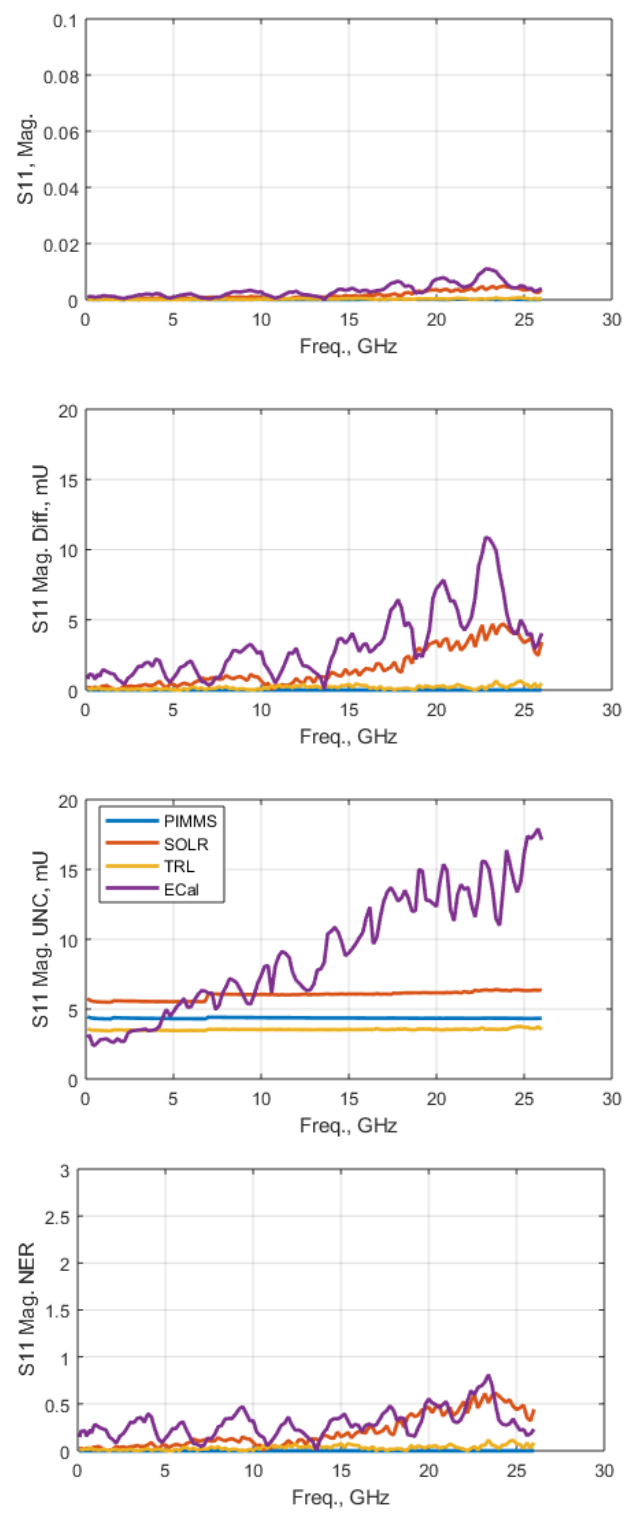

Figure 15: Magnitude of $S_{11}$, difference from PIMMS (Diff), measurement uncertainty (UNC) and NER for the Thru. 

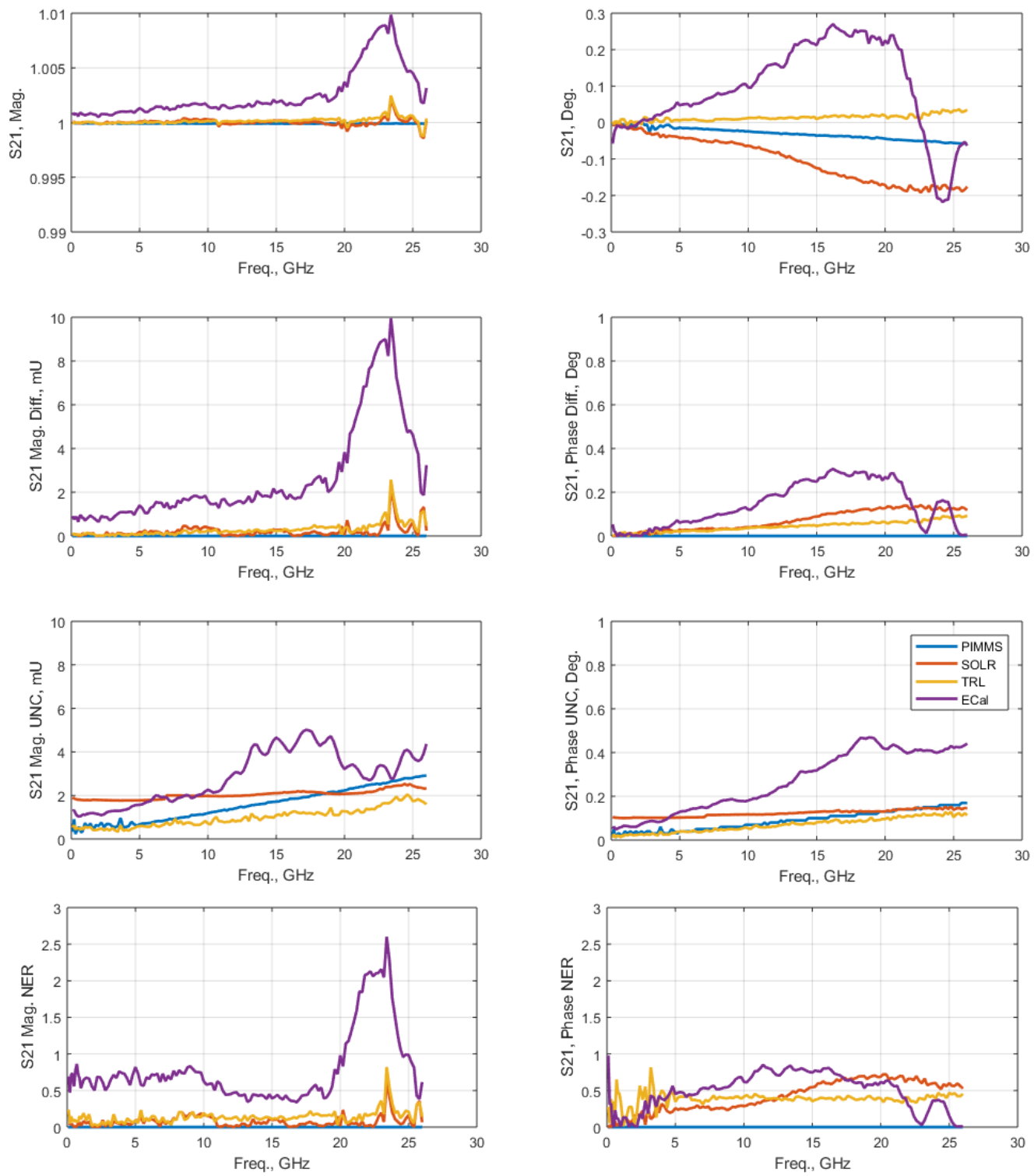

Figure 16: Magnitude and phase of $S_{21}$, difference from PIMMS (Diff), measurement uncertainty (UNC) and NER for the Thru.

\subsubsection{Beatty Line}

A coaxial line with a stepped characteristic impedance going from $50 \Omega$ to $25 \Omega$ and back to $50 \Omega$, also known as a Beatty line [11], was measured as one of the two-port DUTs. A Beatty line is a highly resonant structure and provides a wide range of reflection and transmission coefficients across the frequency range, which is very useful in microwave metrology.

The magnitude and phase of the reflection coefficient $\left(S_{11}\right)$ and transmission coefficient $\left(S_{21}\right)$, the difference relative to the PIMMS measurements, the measurement uncertainties and NER for the Beatty line are plotted in Figure 17 and Figure 18 for all the calibration methods.

Figure 17 shows results for the reflection coefficient $\left(S_{11}\right)$ of the Beatty line. The difference in $S_{11}$ magnitude between the PIMMS measurements and the SOLR and TRL calibrated results is less than 5 $\mathrm{mU}$ but the ECal results differ from the PIMMS results by up to $20 \mathrm{mU}$. The measurement uncertainties 
for the ECal and PIMMS methods are greater than for the SOLR and TRL methods. The uncertainties for PIMMS, SOLR and TRL methods are very similar for frequencies below $10 \mathrm{GHz}$. SOLR and TRL methods show similar and smaller differences from PIMMS compared to the ECal method. The measurement uncertainties of PIMMS are larger than those of VNA-DUO. This may be due to the connection repeatability of the Beatty line, which is not fully accounted for by VNA-DUO (as only Shorts and Loads are used in the repeatability characterisation in VNA-DUO). The measurement uncertainty in the phase of $S_{11}$ varies a lot with frequency as expected for a load of varying impedance. The uncertainty in phase of $S_{11}$ shows peaks at frequencies where the reflection coefficient magnitude is small; a similar situation occurs for the one-port $50 \Omega$ load where the magnitude of $S_{11}$ is very small and the phase is very variable. The measurement uncertainty is larger than the difference from the reference PIMMS measurement for both SOLR and TRL calibrations.
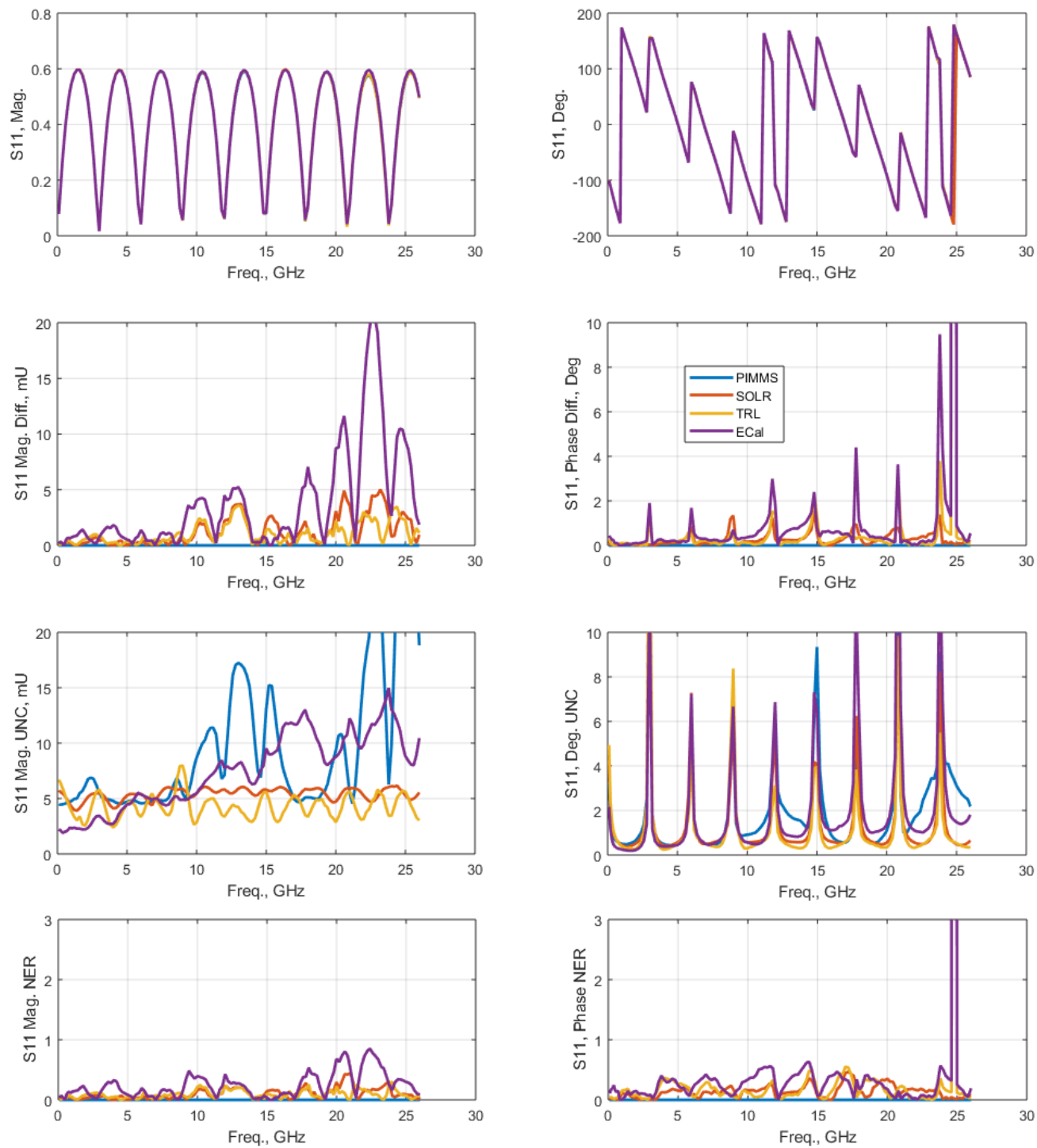

Figure 17: Magnitude and phase of $S_{11}$, difference from PIMMS (Diff), measurement uncertainty (UNC) and NER for the Beatty line.

Figure 18 shows results for the transmission coefficient $\left(S_{21}\right)$ of the Beatty line. The $S_{21}$ results show uncertainties less than $12 \mathrm{mU}$ for magnitude and less than $1^{\circ}$ for phase. The measurement uncertainties 
of $S_{21}$ from the PIMMS and ECal results are larger than those of the SOLR and TRL calibrations. As for the $S_{11}$ measurements, larger uncertainties in $S_{21}$ are expected for the PIMMS measurements due to the connection repeatability of the Beatty Line. The measurement uncertainty in $\mathrm{S}_{21}$ phase above $7 \mathrm{GHz}$ is smaller than the measurement difference from the reference PIMMS measurement for both SOLR and TRL calibrations, which indicates the measurements uncertainties in these methods is unrealistically small. The NER values for the phase of $S_{21}$ for the ECal and TRL calibrations are greater than one. Many other DUTs show NER values greater than one for ECal calibration results, but this is the only DUT which shows NER values greater than one for $S_{21}$ measured with a TRL calibration.
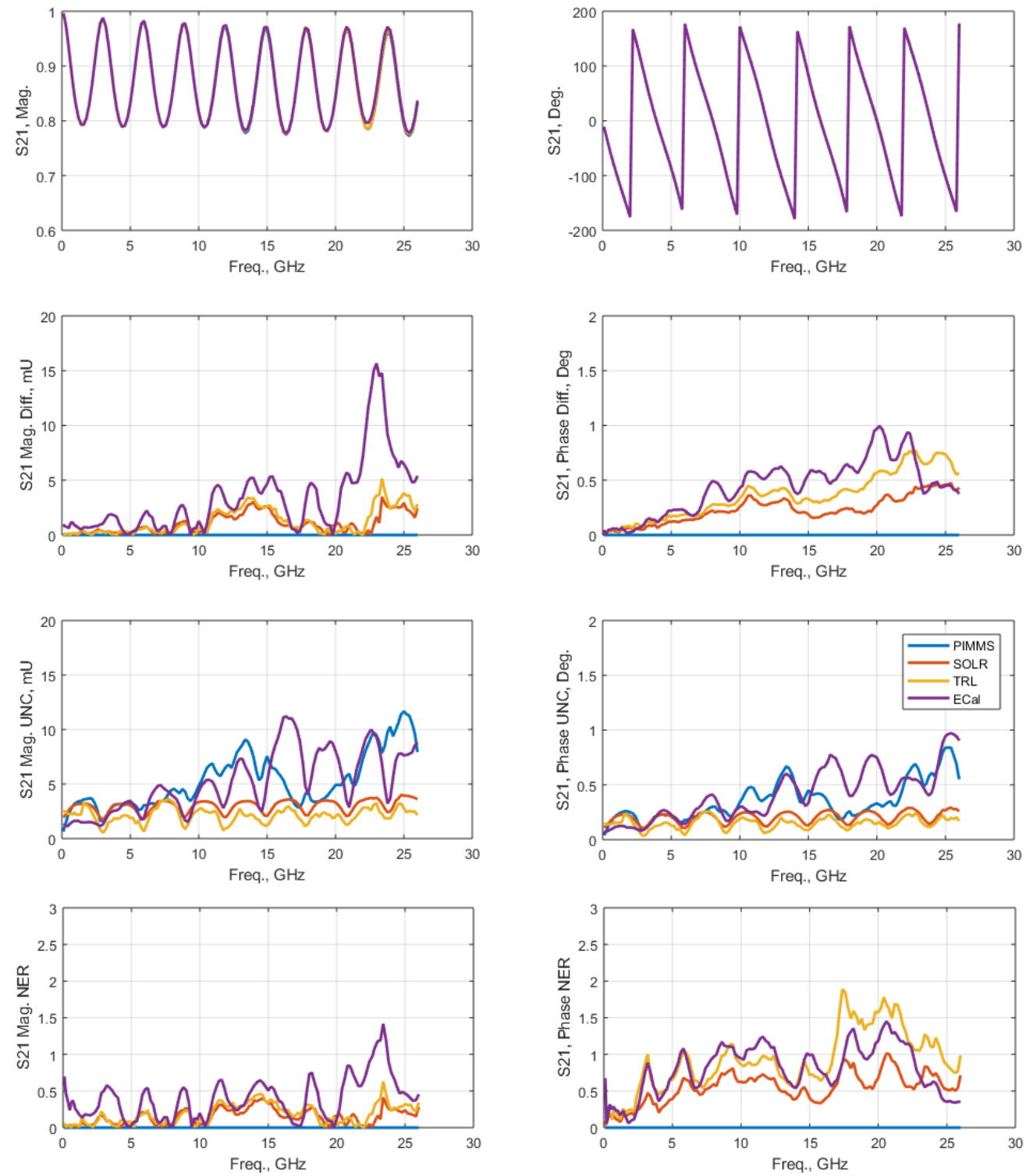

Figure 18: Magnitude and phase of $S_{21}$, difference from PIMMS (Diff), measurement uncertainty (UNC) and NER for the Beatty line.

\subsection{3 $3 \mathrm{~dB}$ Attenuator}

The magnitude of $S_{11}$, the difference from the PIMMS measurements, the measurement uncertainties and NER values for the $3 \mathrm{~dB}$ attenuator for the different calibration methods are plotted in Figure 19. The phase of $S_{11}$ for this and the other attenuators is not plotted because the phase for a very low reflect 
DUT can vary widely, due to the error encompassing the centre of the Smith chart. The maximum difference between results obtained using PIMMS and the other calibration methods is less than $8 \mathrm{mU}$ for the magnitude of $S_{11}$. The measurement uncertainties for the magnitude of $S_{11}$ are less than $8 \mathrm{mU}$. The ECal measurements show larger differences from the PIMMS results and also larger measurement uncertainties compared to the other methods. The uncertainty using SOLR is similar to the PIMMS uncertainty, however, the TRL calibration shows uncertainties that are nearly half that of the PIMMS uncertainties at many frequencies.
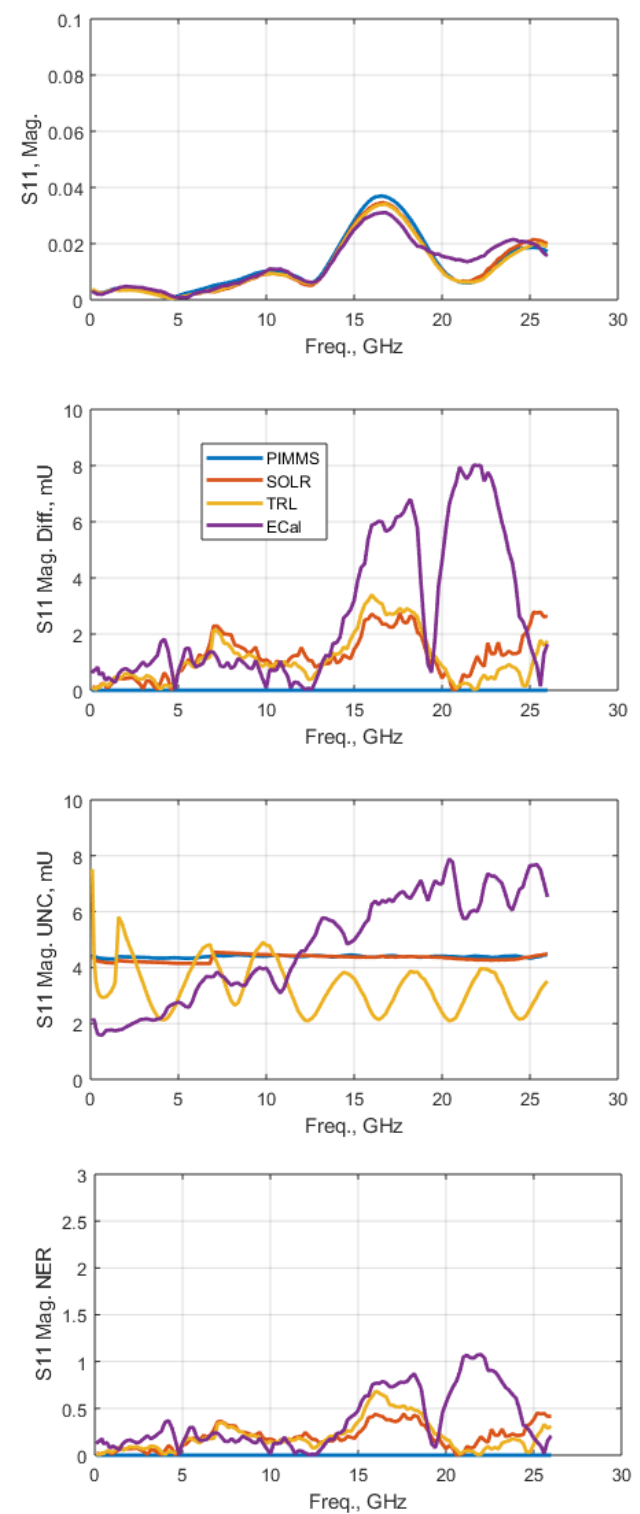

Figure 19: Magnitude of $S_{11}$, difference from PIMMS (Diff), measurement uncertainty (UNC) and NER for the $3 \mathrm{~dB}$ attenuator.

The magnitude and phase of $S_{21}$, the difference from PIMMS, the measurement uncertainties and NER for the $3 \mathrm{~dB}$ attenuator for the different calibration methods are plotted in Figure 20. The maximum $S_{21}$ difference between PIMMS and the other calibration methods can be up to $6 \mathrm{mU}$ for the magnitude and up to $0.5^{\circ}$ for the phase. The $S_{21}$ values show measurement uncertainties less than $4 \mathrm{mU}$ in magnitude and less than $0.5^{\circ}$ in phase. The ECal measurement uncertainties are smaller than the difference from 
the reference PIMMS measurements at some frequencies, which would result in a normalised error ratio greater than 1. The ECal measurements also shows larger differences from the PIMMS results and larger measurement uncertainties compared to the other methods.

The measurement uncertainty is larger than the measurement difference from the reference PIMMS measurement for both SOLR and TRL calibrations. The SOLR uncertainties for $S_{21}$ are closer to the PIMMS uncertainties compared to the TRL uncertainties. ECal again has NER values that are greater than one.
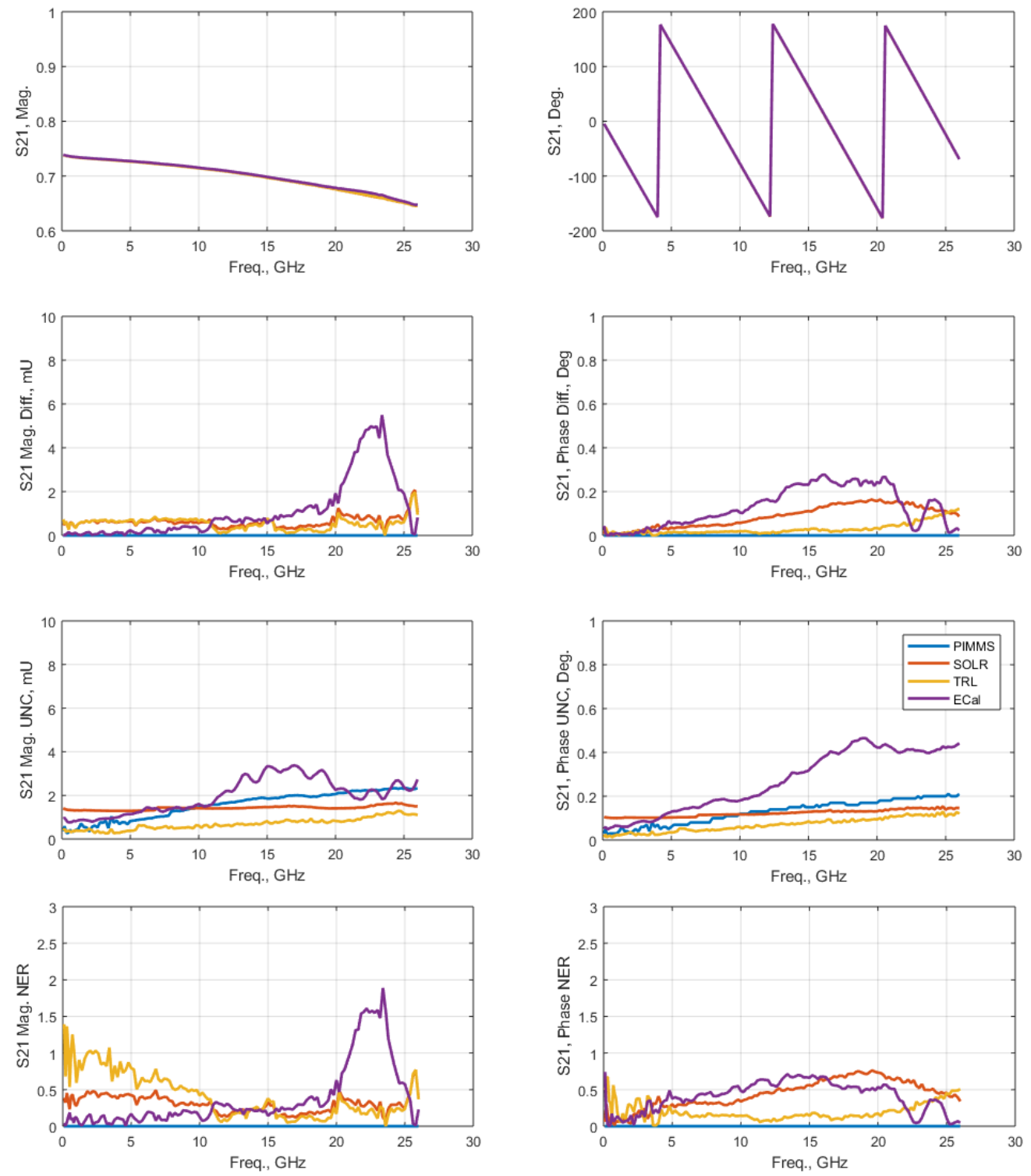

Figure 20: Magnitude and phase of $S_{21}$, difference from PIMMS (Diff), measurement uncertainty (UNC) and NER for the $3 \mathrm{~dB}$ attenuator.

\subsection{4 $20 \mathrm{~dB}$ Attenuator}

The magnitude of $S_{11}$, the difference from the PIMMS measurements, the measurement uncertainties and NER values for the $20 \mathrm{~dB}$ attenuator for the different calibration methods are plotted in Figure 21. The phase of $S_{11}$ for this and the other attenuators is not plotted because the phase for a very low reflect 
DUT can vary widely, due to the error encompassing the centre of the Smith chart. The maximum difference between PIMMS and the SOLR and TRL calibration methods is less than $2.5 \mathrm{mU}$ for the magnitude of $S_{11}$. The measurement uncertainty for the magnitude of $S_{11}$ is less than $5 \mathrm{mU}$.

The ECal measurements shows larger difference from the PIMMS results and larger measurement uncertainties compared to the other methods. The measurement uncertainty of the ECal is also smaller than the difference from PIMMS at some frequencies. The peak in the measurement uncertainty at low frequency in the TRL measurement is due to a limitation in the line calibration standard. The SOLR uncertainties are slightly smaller than the PIMMS uncertainties, but the TRL uncertaintirs are much smaller than the PIMMS uncertainties. The ECal measurements again have NER values that are greater than one.
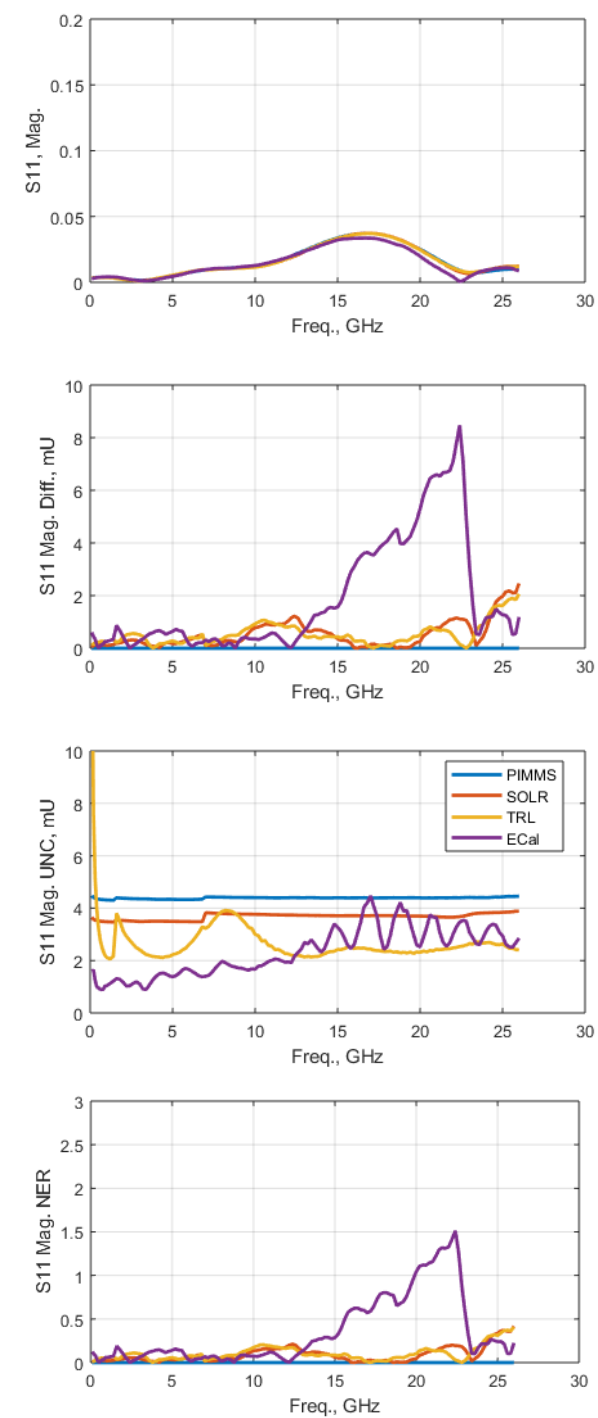

Figure 21: Magnitude of $\mathrm{S}_{11}$, difference from PIMMS (Diff), measurement uncertainty (UNC) and NER for the $20 \mathrm{~dB}$ attenuator.

The magnitude and phase of $S_{21}$, the difference from PIMMS, the measurement uncertainty and NER values for the $3 \mathrm{~dB}$ attenuator for the different calibration methods are plotted in Figure 22. The maximum $S_{21}$ difference between PIMMS and the SOLR and TRL calibration methods can be up to 0.45 $\mathrm{mU}$ for the magnitude and $0.3^{\circ}$ for the phase. The $\mathrm{S}_{21}$ values show measurement uncertainty less than 
$0.5 \mathrm{mU}$ in magnitude and less than $0.5^{\circ}$ in phase. The ECal measurements show larger differences from the PIMMS results and larger measurement uncertainty compared to the other methods. The measurement uncertainty of ECAL is also smaller than the difference from PIMMS at some frequencies.

The measurement uncertainty is larger than the measurement difference from the reference PIMMS measurement for both SOLR and TRL calibrations. Both SOLR and TRL uncertainties are smaller than the PIMMS uncertainties. The TRL uncertainties are unrealistically small.
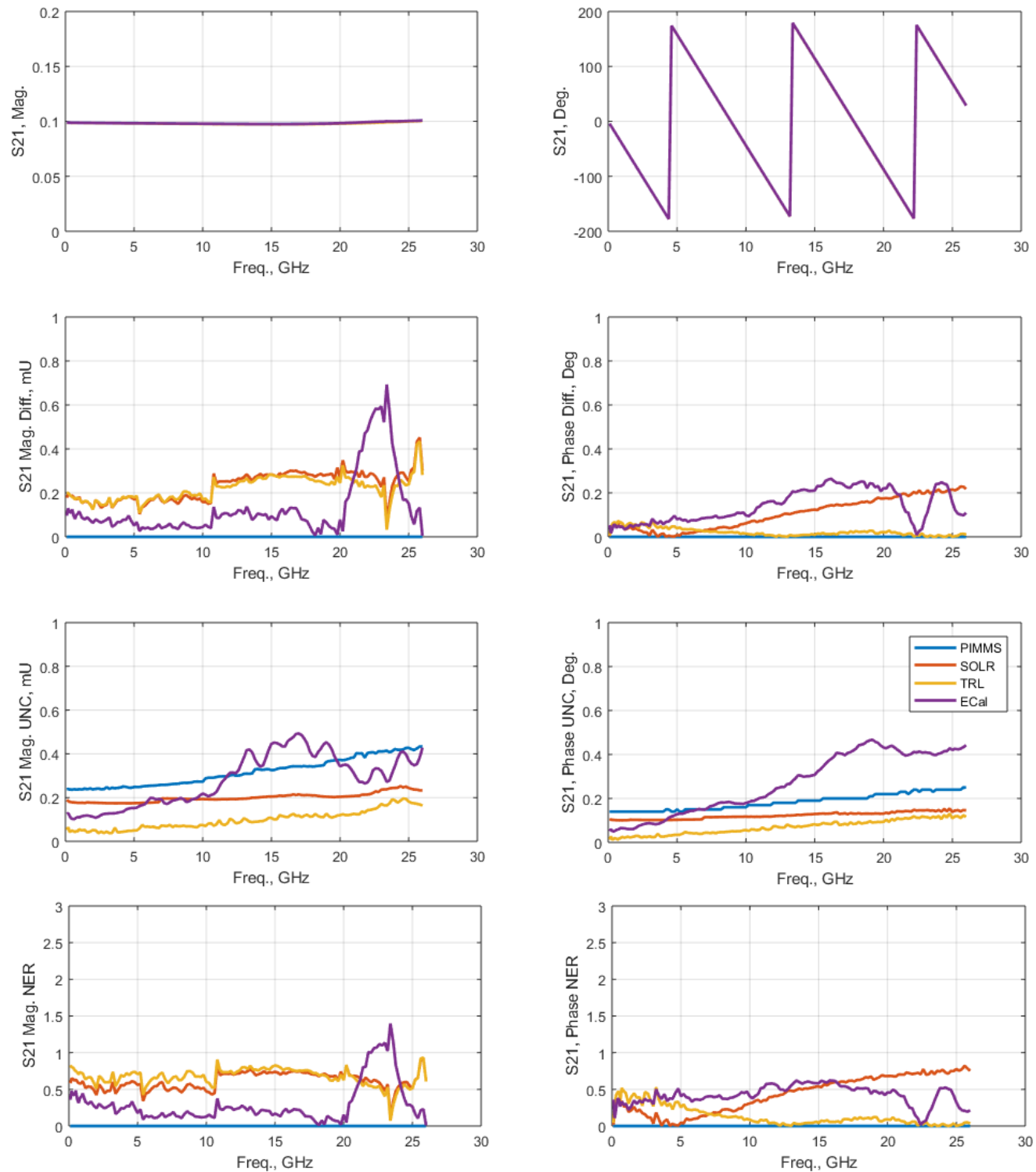

Figure 22: Magnitude and phase of $S_{21}$, difference from PIMMS (Diff), measurement uncertainty (UNC) and NER for the $20 \mathrm{~dB}$ attenuator.

\subsection{5 $40 \mathrm{~dB}$ Attenuator}

The magnitude of $S_{11}$, the difference from the PIMMS measurements, the measurement uncertainty and NER values for the $40 \mathrm{~dB}$ attenuator for the different calibration methods are plotted in Figure 23. The phase of $S_{11}$ for this and the other attenuators is not plotted because the phase for a very low reflect DUT 
can vary widely, due to the error encompassing the centre of the Smith chart. The maximum difference between PIMMS and the SOLR and TRL calibration methods is less than $2 \mathrm{mU}$ for the magnitude of $S_{11}$. The measurement uncertainty for magnitude of $S_{11}$ is less than $5 \mathrm{mU}$. The ECal measurements show larger differences from the PIMMS results and larger measurement uncertainties compared to the other methods. The measurement uncertainty of the ECal is also smaller than the difference from PIMMS at some frequencies. The peak in the measurement uncertainty at low frequency for the TRL measurement is due to a limitation in the line calibration standard.
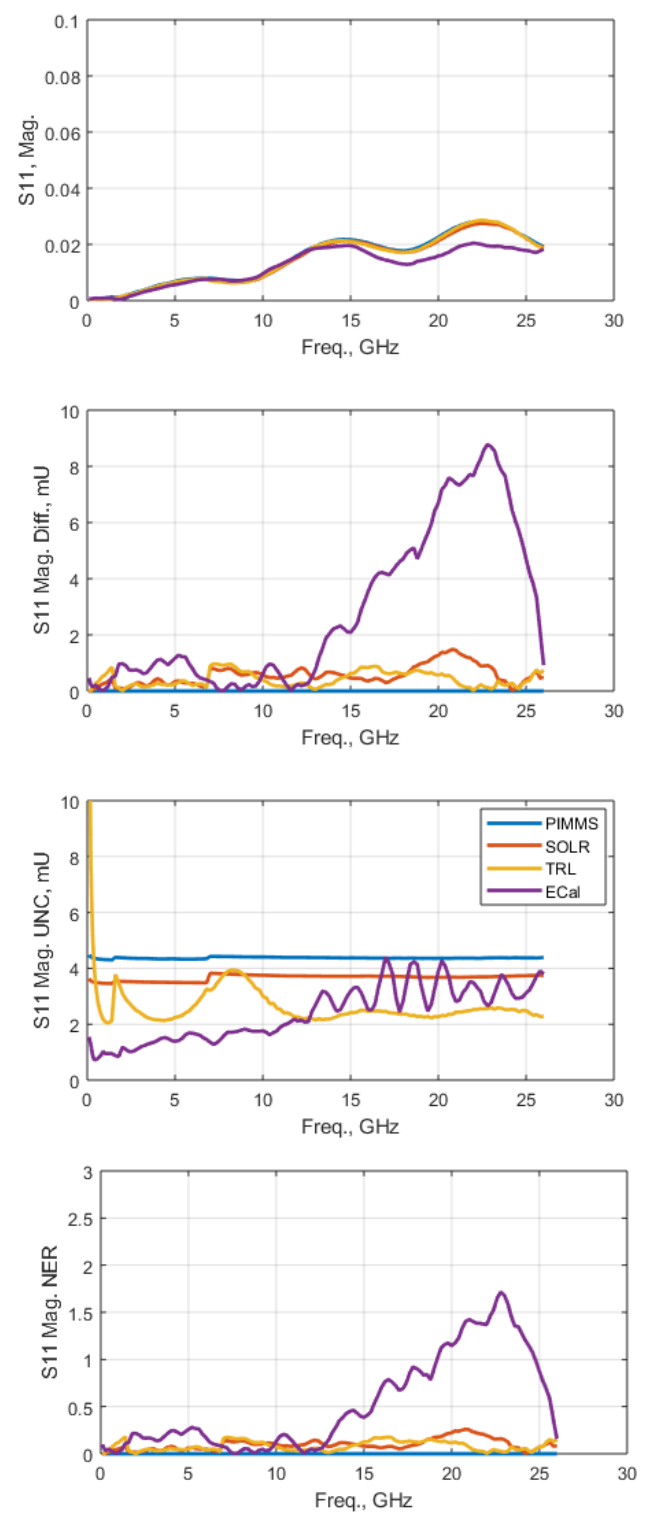

Figure 23: Magnitude of $\mathbf{S}_{11}$, difference from PIMMS (Diff), measurement uncertainty (UNC) and NER for the $40 \mathrm{~dB}$ attenuator.

The magnitude and phase of $S_{21}$, the difference from PIMMS and the measurement uncertainty of the $40 \mathrm{~dB}$ attenuator for the different calibration methods are plotted in Figure 24. The maximum $S_{21}$ difference between PIMMS and the other calibration methods can be up to $0.1 \mathrm{mU}$ for the magnitude and $0.4^{\circ}$ for the phase. The $S_{21}$ values show measurement uncertainty less than $0.1 \mathrm{mU}$ in magnitude and less than $0.5^{\circ}$ in phase. Both SOLR and TRL uncertainties in $S_{21}$ are much smaller than the PIMMS 
uncertainties and the trend shows that the uncertainties in both SOLR and TRL becomes much smaller as the transmission coefficient of the DUT get smaller (as can be seen from the Thru, the $3 \mathrm{~dB}$ attenuator, the $20 \mathrm{~dB}$ attenuator and the $40 \mathrm{~dB}$ attenuator results).
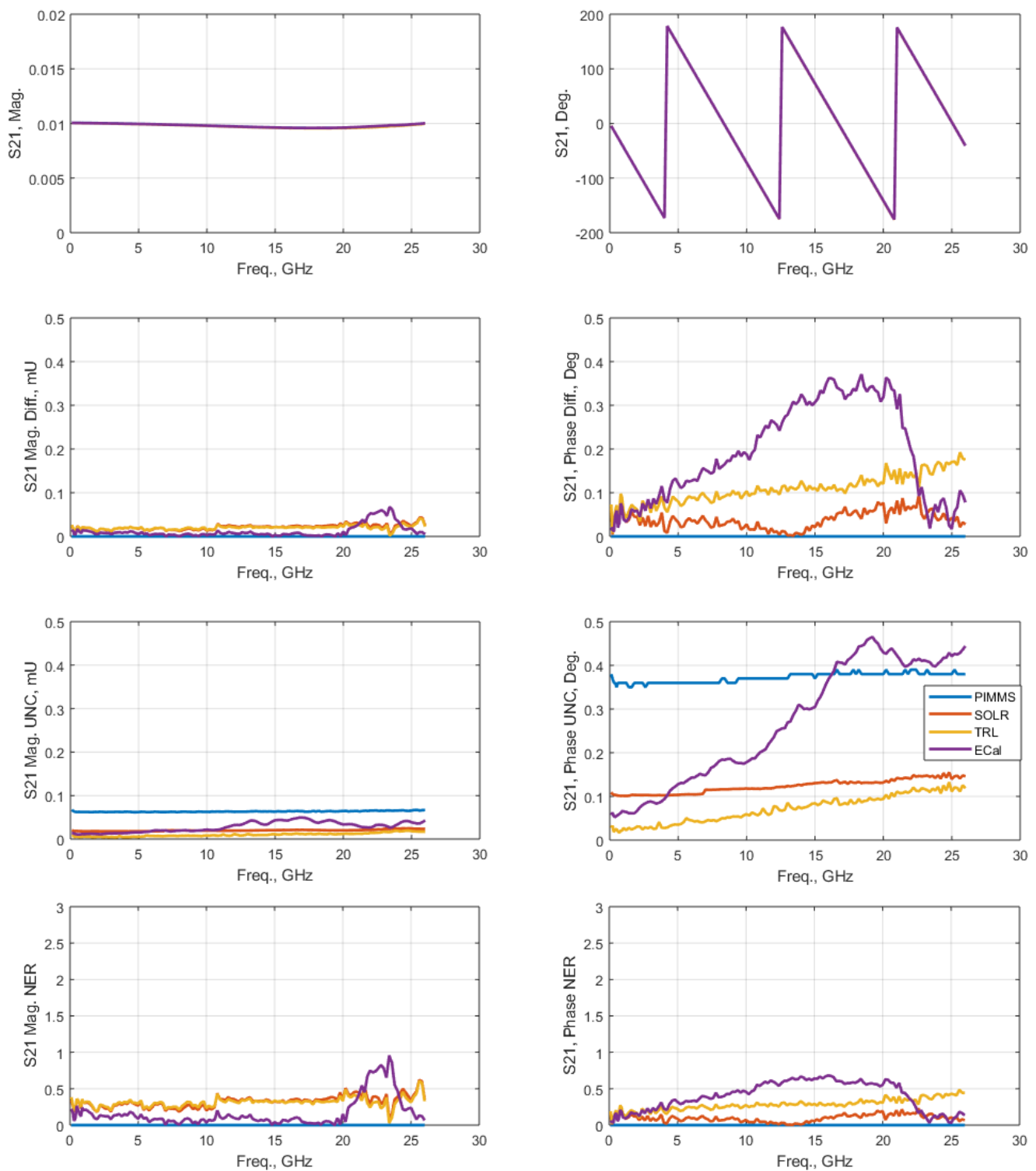

Figure 24: Magnitude and phase of $S_{21}$, difference from PIMMS (Diff), measurement uncertainty (UNC) and NER for the $40 \mathrm{~dB}$ attenuator.

\subsubsection{Summary of Two-Port Device Measurements}

The results for the two-port devices can be summarised by averaging the measurement uncertainty over the measured frequency range. For all two-port devices and all calibration methods, the averaged uncertainties are listed in tables, as follows:

Table 6 - $\mathrm{S}_{11}$, Magnitude average uncertainty 
Table 7 - $\mathrm{S}_{11}$, Phase average uncertainty

Table 8 - $S_{21}$, Magnitude average uncertainty

Table 9- $S_{21}$, Phase average uncertainty

The results can be summarised as follows:

1) The average measurement uncertainty in $S_{11}$ magnitude is between $2 \mathrm{mU}$ and $10 \mathrm{mU}$ for all calibration methods. The measurement uncertainty from PIMMS is around $4.4 \mathrm{mU}$ for all the attenuators and $9.7 \mathrm{mU}$ for the Beatty line. All VNA-DUO calibration methods show decreasing measurement uncertainty with increasing attenuation values. SOLR shows the closest uncertainty to PIMMS.

2) The Beatty line is the only 2-port DUT for which reflection coefficient phase is compared. The average measurement uncertainty for phase of $S_{11}$ ranges between $1.2^{\circ}$ and $2^{\circ}$ for all measurement types. PIMMS has the largest $\mathrm{S}_{11}$ measurement uncertainty of all the methods.

3) The average measurement uncertainty for $S_{21}$ magnitude reduces as the transmission coefficient magnitude decreases as expected. The measurement uncertainty for the SOLR method is closer to PIMMS than that for the TRL method. The PIMMS uncertainties for the Beatty line are much larger than those for the VNA-DUO calibrations.

4) The average measurement uncertainty in $S_{21}$ phase for the SOLR method is again closer to the PIMMS uncertainty than that for the TRL method.

5) The uncertainties in both reflection and transmission coefficients from VNA-DUO SOLR and TRL measurements are of a similar order of magnitude compared to the uncertainties from PIMMS measurements. The SOLR uncertainty is closer to the PIMMS uncertainty than is the TRL uncertainty.

6) The ECal measurements using VNA-DUO show larger measurement uncertainties compared to the SOLR and TRL measurements. The difference in ECal measurements relative to PIMMS measurements is also larger than the measurement uncertainty at some frequencies, resulting in NER values that are greater than one. This has been noted for a large number of DUTs for both reflection and transmission coefficients.

Table 6: Measurement uncertainty in $S_{11}$ magnitude $(\mathrm{mU})$ averaged over frequency for each two-port device and calibration method.

\begin{tabular}{|l|c|c|c|c|}
\hline \multicolumn{1}{|c|}{ DUT } & PIMMS & SOLR & TRL & ECal \\
\hline Thru & 4.36 & 5.98 & 3.54 & 8.83 \\
\hline Beatty & 9.66 & 5.40 & 4.33 & 7.25 \\
\hline 3 dB Attenuator & 4.39 & 4.34 & 3.34 & 4.67 \\
\hline 20 dB Attenuator & 4.39 & 3.68 & 2.76 & 2.29 \\
\hline 40 dB Attenuator & 4.37 & 3.66 & 2.74 & 2.34 \\
\hline
\end{tabular}

Table 7: Measurement uncertainty in $S_{11}$ phase $\left(^{\circ}\right)$ averaged over frequency for each calibration method for the Beatty line.

\begin{tabular}{|l|c|c|c|c|}
\hline DUT & PIMMS & SOLR & TRL & ECal \\
\hline Beatty & 2.00 & 1.37 & 1.17 & 1.89 \\
\hline
\end{tabular}


Table 8: Measurement uncertainty in $\mathrm{S}_{21}$ magnitude $(\mathrm{mU})$ averaged over frequency for each two-port device and calibration method.

\begin{tabular}{|l|c|c|c|c|}
\hline DUT & PIMMS & SOLR & TRL & ECal \\
\hline Thru & 1.65 & 2.04 & 0.97 & 2.81 \\
\hline Beatty & 5.29 & 2.98 & 2.25 & 5.03 \\
\hline 3 dB Attenuator & 1.60 & 1.43 & 0.79 & 1.94 \\
\hline 20 dB Attenuator & 0.31 & 0.20 & 0.11 & 0.28 \\
\hline 40 dB Attenuator & 0.06 & 0.02 & 0.01 & 0.03 \\
\hline
\end{tabular}

Table 9: Measurement uncertainty in $S_{21}$ phase $\left(^{\circ}\right)$ averaged over frequency for each two-port device and calibration method.

\begin{tabular}{|l|c|c|c|c|}
\hline DUT & PIMMS & SOLR & TRL & ECal \\
\hline Thru & 0.09 & 0.12 & 0.07 & 0.26 \\
\hline Beatty & 0.35 & 0.20 & 0.16 & 0.40 \\
\hline 3 dB Attenuator & 0.13 & 0.12 & 0.08 & 0.26 \\
\hline 20 dB Attenuator & 0.18 & 0.12 & 0.08 & 0.26 \\
\hline 40 dB Attenuator & 0.37 & 0.12 & 0.08 & 0.26 \\
\hline
\end{tabular}

\section{INVESTIGATION OF DIFFERENT SOURCES OF UNCERTAINTY}

The S-parameter measurement uncertainty produced using VNA-DUO depends on three components (noise, repeatability and calibration standards), as discussed in Sections 4 and 5. To fully understand the effect of each uncertainty component on the overall uncertainty, an investigation was carried out by measuring DUTs, activating only one uncertainty component at a time. In the VNA-DUO, it is possible to enable/disable any combination of the three uncertainty components.

A Beatty line was measured using SOLR and TRL calibrations in VNA-DUO as follows:

(i) Calibrate VNA-DUO (uncertainty enabled)

(ii) Measure Beatty Line with calibrated VNA and enable uncertainty analyses

(iii) Save S-parameters as *.u2 $\mathrm{p}^{3}$ file with all three uncertainty components enabled

(iv) Save S-parameters as *.u2p file with only the calibration uncertainty component enabled

(v) Save S-parameters as *.u2p file with only the noise uncertainty component enabled

(vi) Save S-parameters as *.u2p file with only the repeatability uncertainty component enabled

This procedure was carried for both SOLR and TRL calibrations.

Figure 25 shows the measurement uncertainty for the reflection and transmission coefficients of a Beatty line measured with the VNA calibrated using TRL, and Figure 26 shows the same except using a SOLR calibration. The results from both calibrations show that the contribution from the noise source is minimal (i.e. is close to zero) for both reflection and transmission coefficients. The connector and cable repeatability sources of uncertainty are the second largest contributor in both types of calibrations. The uncertainty contribution due to the calibration standards is the most significant (i.e. dominant) source of uncertainty for both magnitude and phase of the S-parameters for both TRL and SOLR calibrations.

Since the overall measurement uncertainty is dominated by the uncertainty in the calibration standards, it is important to have the calibration standards characterised accurately - e.g. by a National Metrology

\footnotetext{
${ }^{3}$ See the Appendix for file format details
} 
Institute such as NPL. The calibration standards used in the investigation described in this report were characterised using NPL's primary national standard facility for S-parameter measurement (i.e. PIMMS) and is traceable to fundamental SI units (i.e. the metre and the second).
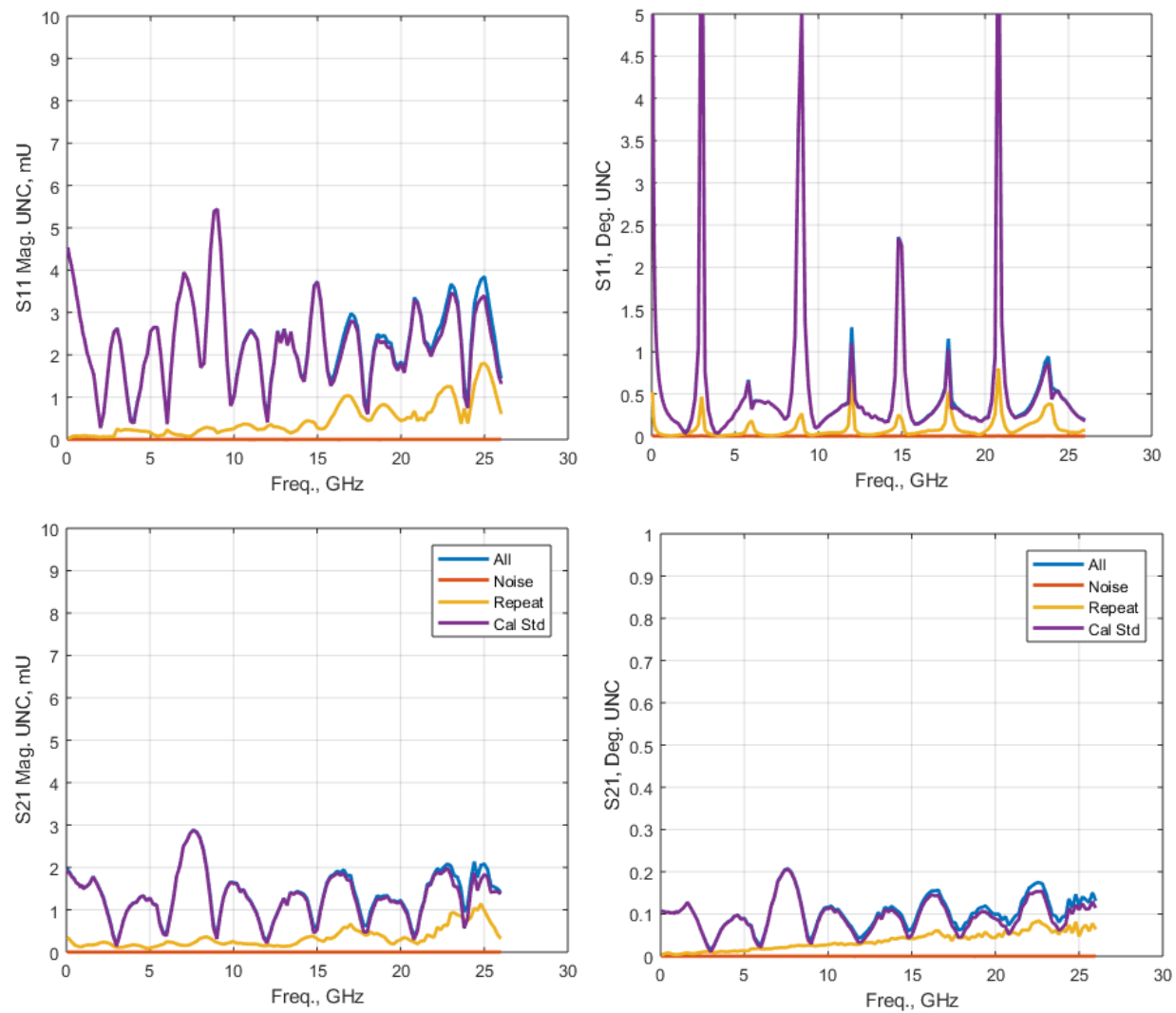

Figure 25: Measurement uncertainty contributions due to noise, repeatability and calibration standards for a Beatty line measured using TRL calibration. 

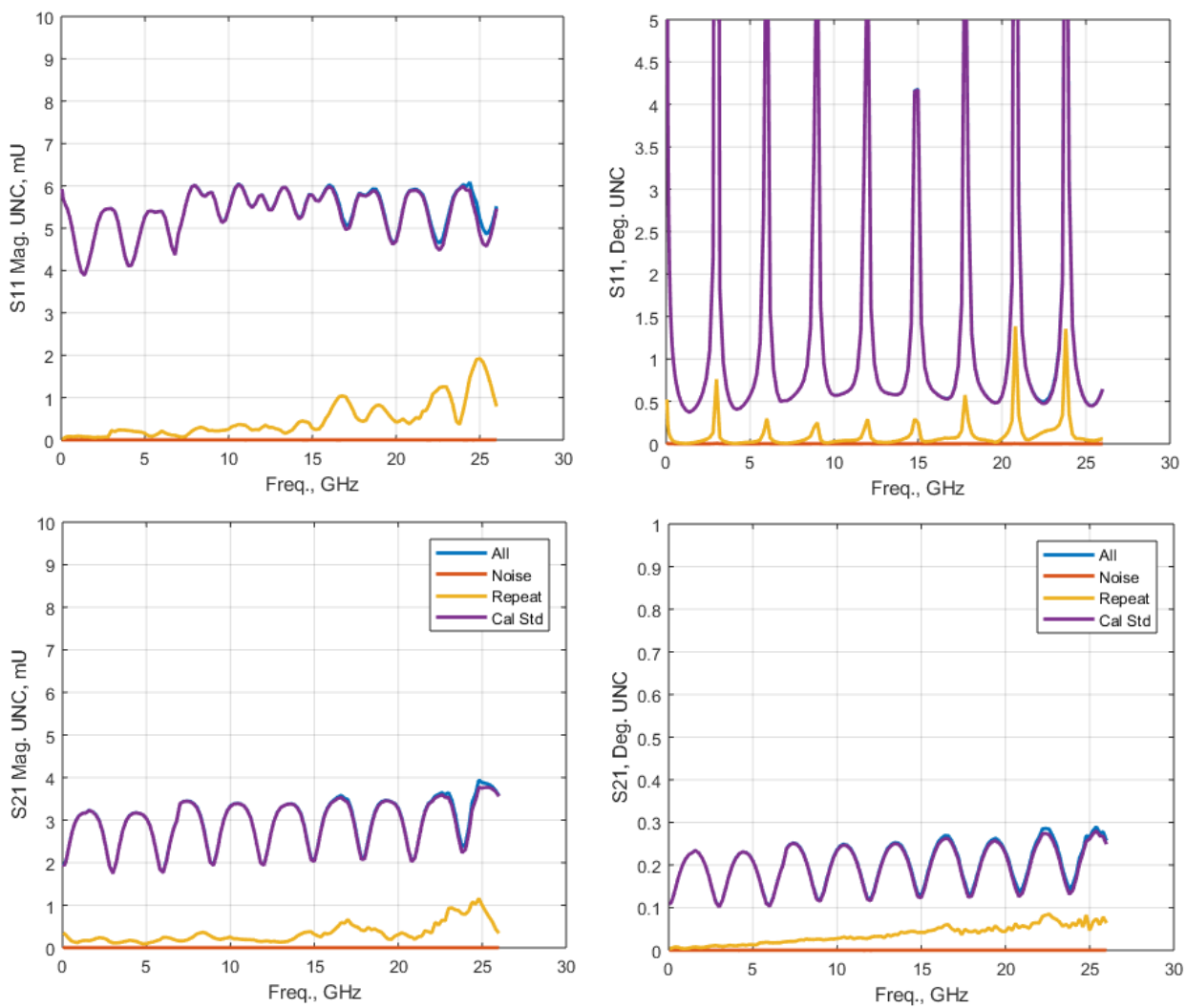

Figure 26: Measurement uncertainty contributions due to noise, repeatability and calibration standards for a Beatty line measured using SOLR calibration.

\section{CONTRIBUTION OF EACH CALIBRATION STANDARD TO THE MEASUREMENT UNCERTAINTY}

In Section 8, the uncertainty due to the three main sources of uncertainty was investigated and it was found that the uncertainty due to the calibration standards is the most significant contributor to the overall uncertainty. For SOLR and TRL calibrations, the uncertainty due to the calibration standards is due to uncertainty from each individual standard used during the calibration. This section presents an investigation into the contribution to the uncertainty due to each of the standards used for TRL and SOLR calibrations.

TRL calibration uses Thru, Reflect and Line standards and SOLR uses Short, Open, Load and reciprocalThru calibration standards. For each calibration standard, two definition files (*.dsd $\left.{ }^{4}\right)$ were created; (i) with uncertainty data from PIMMS, and (ii) with all uncertainty data set to "Zero". Nine VNA-DUO calibrations were carried out with calibration definitions as follows:

i. TRL calibration "Thru only" - using only the uncertainty information for the Thru standard; the uncertainty for the other standards was set to zero

ii. TRL calibration "Short only" - using only the uncertainty information for the Reflect (i.e. shortcircuit); the uncertainty for the other standards was set to zero

iii. TRL calibration "Line only" - using only the uncertainty information for the Line standard; the uncertainty for the other standards was set to zero

\footnotetext{
${ }^{4}$ See the Appendix for file format details
} 
iv. TRL calibration "all standards" - using the uncertainty information for all the calibration standards

v. SOLR calibration "Load only" - using only the uncertainty information for the Load; the uncertainty for the other standards was set to zero

vi. SOLR calibration "Open only" - using only the uncertainty information for the Open; the uncertainty for the other standards was set to zero

vii. SOLR calibration "Short only" - using only the uncertainty information for the Short; the uncertainty for the other standards was set to zero

viii. SOLR calibration "Thru only" - using only the uncertainty information for the Thru; the uncertainty for the other standards was set to zero

ix. SOLR calibration "all standards" - using the uncertainty information for all the calibration standards

All the above calibrations were saved to the VNA firmware. The uncalibrated (i.e. raw) S-parameters of a Beatty Line were measured using the VNA. The VNA trace was subsequently put on "hold" so that the uncorrected data was saved within the VNA. All the above (nine) calibrations stored in the VNA firmware were then applied, one at a time, to the saved uncorrected data so that the corrected Sparameters (as *.u2p files) could be generated.

For this investigation, only one of the TRL Line standards was used during calibration, to help simplify the investigation. The $16.17 \mathrm{~mm}$ line was chosen for the TRL calibration, resulting in a reduced frequency range of $1.5 \mathrm{GHz}$ and $7 \mathrm{GHz}$ (i.e. the useable range for this line as a standard with a TRL calibration). The S-parameter measurement uncertainties for the four TRL calibrations labelled (a) to (d), above, for measurements of the Beatty Line are plotted in Figure 27. Similarly, the S-parameter measurement uncertainties for the five SOLR calibrations labelled (e) to (i), above, for measurements of the Beatty Line are plotted in Figure 28.

The TRL calibration using only uncertainty due to the short-circuit (i.e. calibration (b), above) produces the lowest uncertainty. The TRL calibration using only uncertainty due to the Thru (i.e. calibration (a), above), produces uncertainties that are very similar to measurements using uncertainty due to all the standards (i.e. calibration (d), above). The Line calibration standard (i.e. calibration (c), above) does have some effect on the overall uncertainty - however, the overall uncertainty is dominated by the uncertainty in the Thru standard. Therefore, the following conclusions can be drawn from this investigation into the TRL calibration:

a) The Thru standard is the most significant source of uncertainty

b) The Line standard is the second most significant source of uncertainty

c) The Short (Reflect) standard is the least significant source of uncertainty 

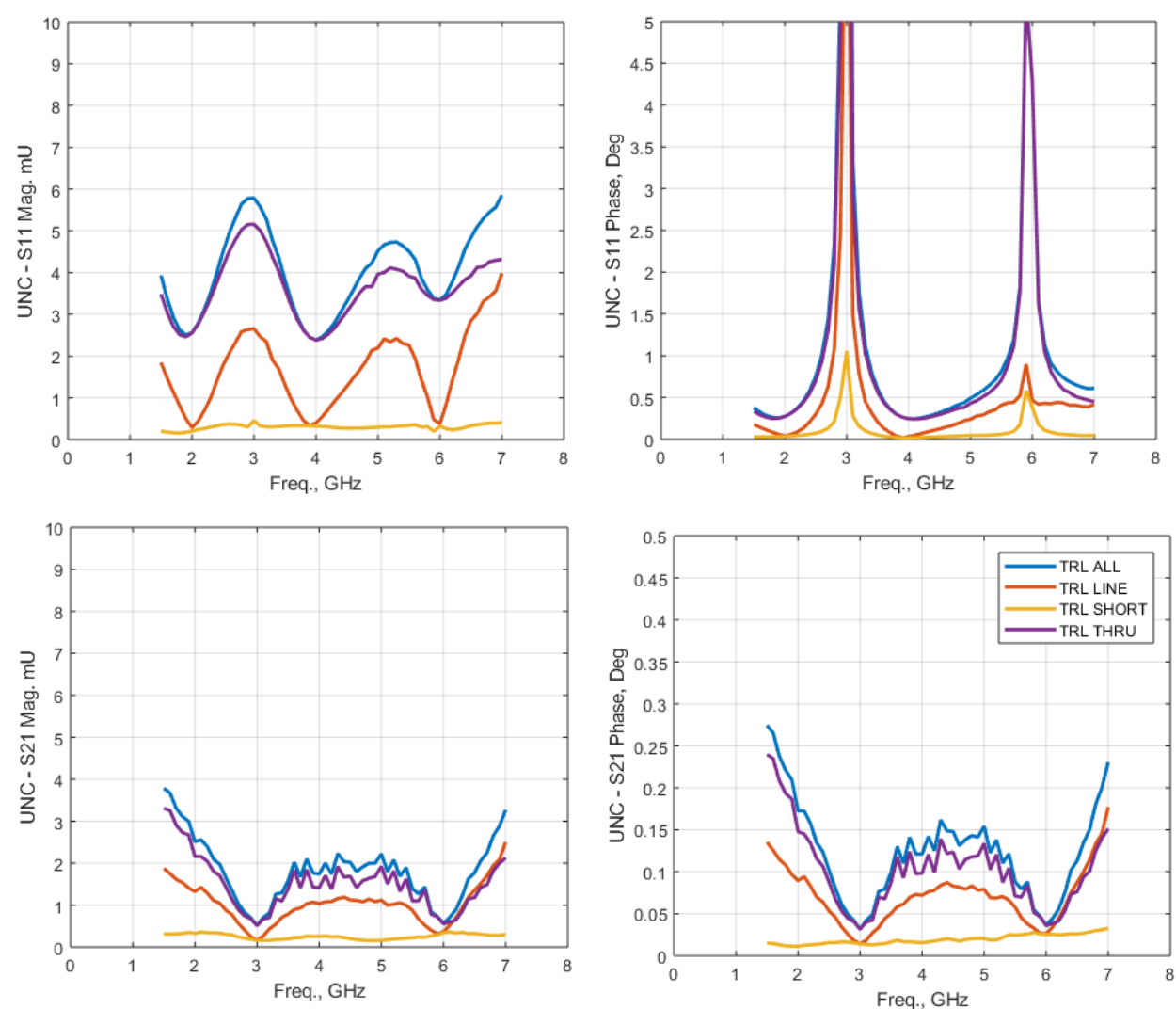

Figure 27: Contributions to the measurement uncertainty due to different calibration standards for measurements of a Beatty line, measured using a TRL calibration.

Contributions to the measurement uncertainty due to different calibration standards for measurements of a Beatty line in the SOLR calibration is plotted in Figure 28. The SOLR calibration using only uncertainty due to the thru (i.e. calibration (h), above) produces the lowest uncertainty. The SOLR calibration using only uncertainty due to the load (i.e. calibration (e), above), produces uncertainties that are very similar to measurements using uncertainty due to all the standards (i.e. calibration (d), above) for $S_{11}$. The load, short and open calibration standards (i.e. calibration (e, $f$ and $g$ ), above) have similar effect on the overall uncertainty for $S_{21}$. Therefore, the following conclusions can be drawn from this investigation into the SOLR calibration:

a) The Load standard is the most significant source of uncertainty

b) The Short and Open standards make similar contributions to the uncertainty but smaller than that of the Load standard

c) The Thru standard is the least significant source of uncertainty 

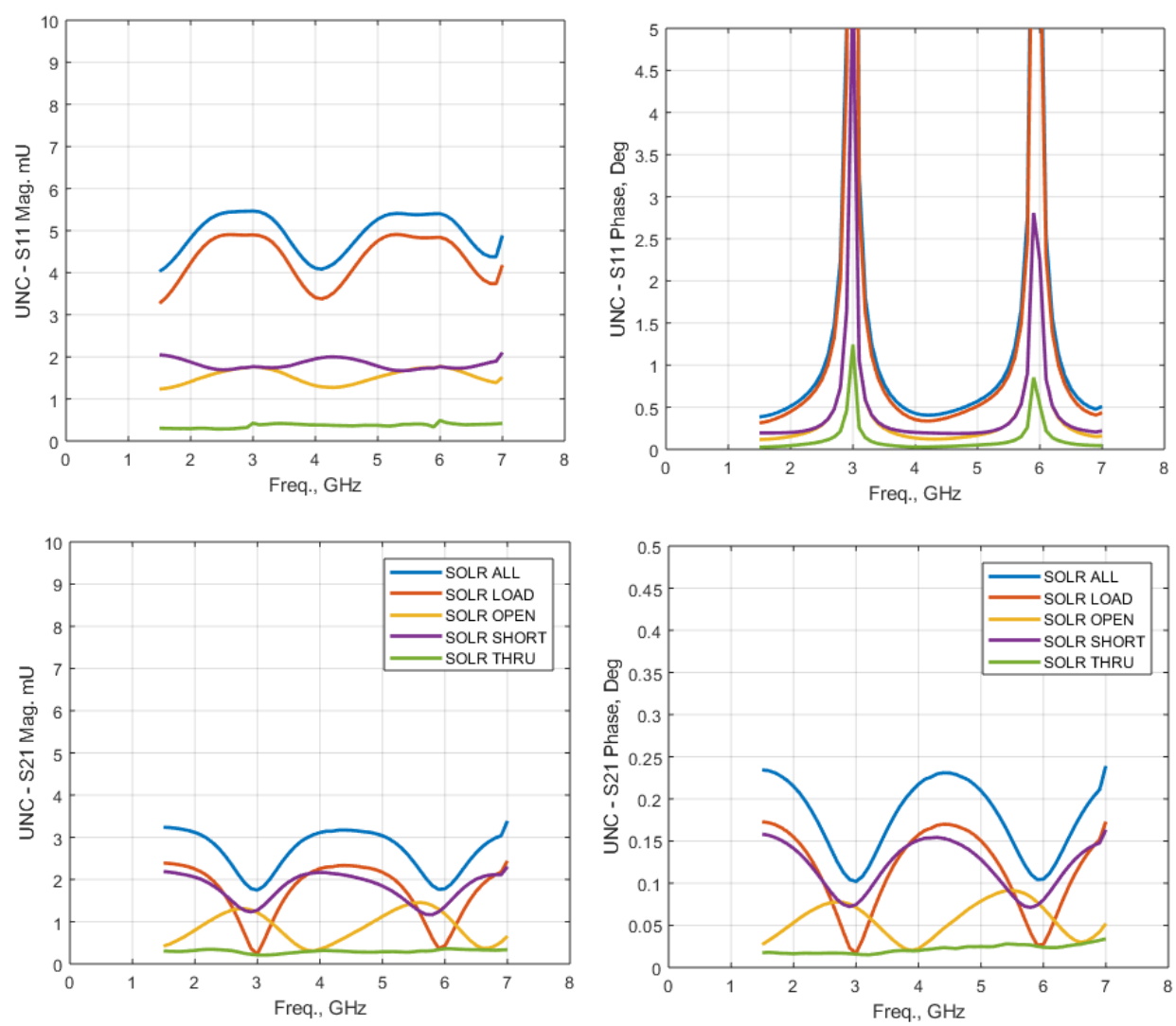

Figure 28: Contributions to the measurement uncertainty due to different calibration standards for measurements of a Beatty line, measured using a SOLR calibration. 


\section{SUMMARY \& CONCLUSIONS}

This report has investigated VNA-DUO installed on a Keysight Technologies PNA-X VNA. VNA-DUO offers significant advantages when determining S-parameter measurement uncertainty. It can considerably reduce the device characterisation time as the associated uncertainties are displayed in real-time with the measurements.

The fundamental principles and all the sources of the S-parameter measurement uncertainty considered in VNA-DUO have been discussed.

VNA-DUO has been evaluated using seven one-port DUTs (two offset short-circuits, two offset opencircuits, two reflection check standards and a $50 \Omega$ termination) and five two-port DUTs (a thru, a Beatty line, a $3 \mathrm{~dB}$ attenuator, a $20 \mathrm{~dB}$ attenuator and a $40 \mathrm{~dB}$ attenuator). Three calibration types were investigated: TRL, SOLR, and ECal. The measurement uncertainties using these calibrations were compared with NPL's PIMMS measurements of the same DUTs. The analysis was performed separately for both the magnitude and phase components of the measured complex-valued reflection and transmission coefficients.

The investigation included the following:

A) Comparison of mechanically defined, manufacturer defined and PIMMS characterized calibration standards.

B) VNA-DUO measurement uncertainty for TRL, SOLR and ECal calibrations compared with PIMMS.

C) A comparison of the uncertainty contributions due to noise, repeatability and calibration standards in TRL and SOLR calibration measurements made with VNA-DUO.

D) A comparison of the impact on measurement uncertainty due to each calibration standard used in TRL and SOLR calibrations made with VNA-DUO.

The following conclusions can be drawn from the work described in this report:

i) The S-parameter measurement uncertainties evaluated by VNA-DUO when the calibration standard definitions supplied by the manufacturer (i.e. Keysight) are used, are much larger than the uncertainties evaluated using the reference PIMMS method. When mechanical calibration standard definitions (based on dimensions and tolerances) are used, the measurement uncertainties evaluated using VNA-DUO are much smaller than those evaluated using the reference PIMMS method. When the VNA-DUO calibration standards are characterised using PIMMS, the uncertainties evaluated by VNA-DUO show the closest agreement with the uncertainties evaluated using the reference PIMMS method. Thus, more realistic uncertainties are achieved from VNA-DUO when the calibration standards are characterised by PIMMS (or by some other primary S-parameter measurement system).

ii) The investigation, described in Section 8, into the three different sources of uncertainty considered in VNA-DUO shows that the calibration standards are the major source of uncertainty in the S-parameter measurements. Therefore, accurate characterisation of the calibration standards (in terms of both S-parameter values and their uncertainties) is very important to achieve reliable measurement results using VNA-DUO. Connector repeatability can also make a significant contribution to the uncertainty in S-parameter measurements, but the noise floor contribution to the uncertainty is negligible compared to the other two sources of uncertainty.

iii) VNA-DUO does not include uncertainty due to the connector repeatability of the actual DUT and so can produce smaller S-parameter uncertainties than PIMMS. This is particularly evident for more elaborate DUTs, such as the Beatty line.

iv) In the TRL calibration, the thru standard is the most significant source of uncertainty, followed by the line standard. The short (reflect) standard is the least significant source of uncertainty. 
v) In the SOLR calibration, the Load standard is the most significant source of uncertainty, followed by the Short and the Open standards. The Thru standard is the least significant source of uncertainty.

vi) Of the three calibration methods investigated using VNA-DUO (SOLR, TRL and ECal), the SOLR calibration shows uncertainties that are closest to the PIMMS uncertainties (when the calibration standards are characterized using PIMMS). The SOLR measurement results also shows the closest agreement with the PIMMS measurement results - the NER values for the SOLR measurements relative to PIMMS are less than unity for all DUTs and all S-parameters.

vii) The TRL calibration in VNA-DUO produces smaller measurement uncertainties than PIMMS. The uncertainties evaluated by VNA-DUO for TRL calibration are therefore considered to be unrealistically small. The NER values show some degree of inconsistency between VNA-DUO TRL the and PIMMS measurements.

viii) The ECal calibration in VNA-DUO, which uses calibration standard definitions supplied by the manufacturer, shows much larger deviations from the reference PIMMS measurements compared to the other calibration methods investigated in VNA-DUO (i.e. SOLR and TRL). It also shows relatively large uncertainties. The NER values show considerable inconsistency between VNA-DUO ECal and the PIMMS measurements.

ix) VNA-DUO does not include uncertainties due to the connector repeatability of the actual DUT nor uncertainties due to any non-linearity in the VNA detectors. Non-linearity effects may be significant when measuring DUTs with large values of attenuation.

This investigation has shown that primary level calibration accuracy, from National Metrology Institutes (NMIs) such as NPL, can be transferred to other VNA end-users (e.g. in industry) using VNA-DUO. This could provide more efficient, easy-to-implement uncertainty assessments for all VNA end-users.

It is considered that VNA-DUO is a very useful tool to give indicative information about S-parameter measurement uncertainty in real-time and can provide realistic estimates for S-parameter uncertainty, under certain conditions. These conditions were found to be when the VNA-DUO standards have been characterised using PIMMS measurements and these standards are used subsequently to perform an SOLR calibration. It is therefore recommended that these conditions are followed when using the VNADUO to achieve realistic uncertainties.

For the most accurate S-parameter measurements, it is still recommended to access measurement capabilities at NMIs (such as NPL, NIST, PTB, etc).

\section{ACKNOWLEDGMENTS}

The work described in this report was funded through the European Metrology Programme for Innovation and Research (EMPIR) Project JRP 16ENG04 ADVENT 'Metrology for advanced energysaving technology in next-generation electronic applications.' The EMPIR is jointly funded by the EMPIR participating countries within EURAMET and the European Union. The work was also supported by the National Measurement Sustem (NMS) Programme of the UK Government's Department for Business, Energy and Industrial Stretegy (BEIS). 


\section{REFERENCES}

[1] Evaluation of measurement data - Guide to the expression of uncertainty in measurement, GUM 1995, with minor modifications, JCGM 100:2008 https://www.bipm.org/en/publications/guides/.

[2] Evaluation of measurement data - Supplement 2 to the Guide to the expression of uncertainty in measurement - Extension to any number of output quantities, JCGM 102:2011 https://www.bipm.org/en/publications/guides/.

[3] Guidelines on the Evaluation of Vector Network Analysers (VNA), EURAMET Calibration Guide No. 12, Version 3.0 (03/2018) https://www.euramet.org/publications-media-centre/calibrationguidelines/.

[4] NIST Microwave Uncertainty Framework https://www.nist.gov/services-resources/software/wafer-calibration-software

[5] METAS VNA Tools https://www.metas.ch/metas/en/home/fabe/hochfrequenz/vna-tools.html

[6] N M Ridler, "A review of existing national measurement standards for RF and microwave parameters in the UK", IEE Colloquium Digest, No 99/008, pp 6/1 - 6/6, February 1999.

[7] "VNA Measurement Uncertainty - Real Time S-Parameter and Power Uncertainty", Keysight Technologies Application Note - 5992-3595EN, June 26, 2019.

[8] "IEEE Standard for Precision Coaxial Connectors (DC to $110 \mathrm{GHz}$ )", IEEE Std 287-2007.

[9] M Garelli and A Ferrero, "A unified theory for S-parameter uncertainty evaluation", IEEE Transactions on Microwave Theory and Techniques, Vol 60, No 12, pp 3844 - 3855, Dec. 2012.

[10] D Singh, M J Salter and N M Ridler, "Comparison Of Vector Network Analyser (VNA) Calibration Techniques At Microwave Frequencies," NPL Report TQE 14, National Physical Laboratory, Teddington, UK, Dec 2019.

[11] R W Beatty, "Calculated and Measured S11, S21, and group delay for simple types of coaxial and rectangular waveguide 2-port standards", NBS Technical Note 657, December 1974. 


\section{APPENDIX}

This appendix lists the file formats used for S-parameter data and calibration standard definitions in PIMMS and VNA-DUO. The PIMMS tool from NPL saves the transmission coefficients of the DUT in *T.RID format and a typical file format is shown in 1). PIMMS saves the reflection coefficients of the DUT in *R.RID format as shown in 2).

VNA-DUO saves the measured S-parameters of the DUT with uncertainty as a *.u2p file shown in 3) and it uses a $*$.dsd file format shown in 4 ) for the calibration standard definitions.

1) PIMMS transmission file format:

UNCERT (T) file: C:Ipimmsldb\Advent_PIMMS_V_Option15।3T.RID

Produced by program: PIMMS Version 6.1.6 January 2010

Operator: James Skinner on 15/01/2019 at 11:57:54

20dB Attenuator, SN 00122 (t)

$\begin{array}{ll}\text { S21 } & \text { S12 }\end{array}$

Frequency

$\begin{array}{lllllllll}(\mathrm{GHz}) & \text { Real } & \text { Imaginary } & \text { Uncertainty } & \mathrm{K} & \text { Real } & \text { Imaginary } & \text { Uncertainty } & \mathrm{K} \\ 0.1 & 3.22 \mathrm{e}-3 & 2.22 \mathrm{e}-4 & 4.47 \mathrm{e}-3 & 2.50 \mathrm{e} 0 & 4.52 \mathrm{e}-3 & -1.97 \mathrm{e}-4 & 4.43 \mathrm{e}-3 & 2.50 \mathrm{e} 0 \\ 0.2 & 3.45 \mathrm{e}-3 & 2.89 \mathrm{e}-4 & 4.40 \mathrm{e}-3 & 2.50 \mathrm{e} 0 & 4.56 \mathrm{e}-3 & -4.59 \mathrm{e}-4 & 4.40 \mathrm{e}-3 & 2.50 \mathrm{e} 0\end{array}$

2) PIMMS reflection file format:

UNCERT (R) file: C:Ipimmsldb\Advent_PIMMS_V_Option15।3R.RID

Produced by program: PIMMS Version 6.1.6 January 2010

Operator: James Skinner on 15/01/2019 at 11:57:54

20dB Attenuator, SN 00122 (r)

S11

Frequency

$(\mathrm{GHz})$ Real Imaginary Uncertainty $\mathrm{K}$

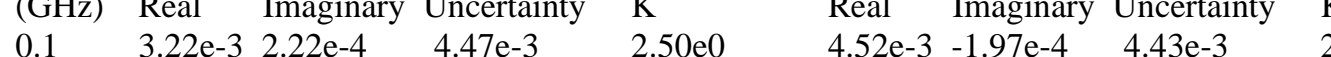

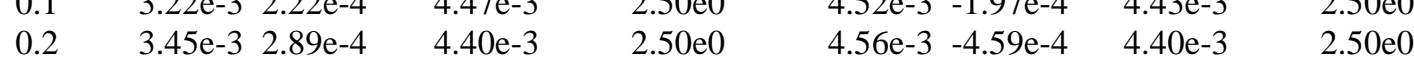

3) VNA-DUO *.u2p file format

!CSV A.02.01

!Keysight Technologies,N5247B,US51370340,A.13.95.07

!Date: Thursday, June 18, 2020 17:04:22

!Uncertainty data: S-parameter standard deviation

!Coverage factor: $\mathrm{k}=1$

Freq(Hz), S1_1 mag (linear), S1_1 phase (deg), S1_2 mag (linear), S1_2 phase (deg), S2_1 mag (linear), S2_1 phase (deg), S2_2 mag (linear), S2_2 phase (deg), US1_1 mag (linear), US1_1 phase (deg), US1_2 mag (linear), US1_2 phase (deg), US2_1 mag (linear), US2_1 phase (deg), US2_2 mag (linear), US2_2 phase (deg)

$\begin{array}{lllll}1.000000000000 \mathrm{e}+008, & 1.590457558632 \mathrm{e}-001, & 1.677574236765 \mathrm{e}+002, & 8.343093213625 \mathrm{e}-005,- \\ 1.295378297605 \mathrm{e}+002, & 5.200302124023 \mathrm{e}+000, & 1.700810950132 \mathrm{e}+002, & 1.922370344400 \mathrm{e}-001, \\ 1.568098735314 \mathrm{e}+002, & 7.113435565954 \mathrm{e}-004, & 1.650748245391 \mathrm{e}-001, & 4.875919066570 \mathrm{e}-005, \\ 3.348511241705 \mathrm{e}+001, & 7.423406759732 \mathrm{e}-003, & 7.325244807219 \mathrm{e}-002, & 6.068102727219 \mathrm{e}-004, \\ 1.335688829549 \mathrm{e}-001 & & & \\ 2.000000000000 \mathrm{e}+008, & 1.566576063633 \mathrm{e}-001, & 1.564112636914 \mathrm{e}+002, & 1.029279083014 \mathrm{e}-004, \\ 1.096497942183 \mathrm{e}+002, & 5.108454227448 \mathrm{e}+000, & 1.578636760976 \mathrm{e}+002, & 1.969771683216 \mathrm{e}-001, \\ 1.477744758255 \mathrm{e}+002, & 6.966169841976 \mathrm{e}-004, & 1.917847922886 \mathrm{e}-001, & 4.338692831685 \mathrm{e}-005, \\ 2.415171667640 \mathrm{e}+001, & 7.555920623529 \mathrm{e}-003, & 7.872450756952 \mathrm{e}-002, & 6.483276313791 \mathrm{e}-004, \\ 1.378618308025 \mathrm{e}-001 & & & & \end{array}$




\section{$3.000000000000 \mathrm{e}+008$}

4) VNA-DUO *.dsd file format

!MMS4 Definition Standard Data

!Filename:

C:IUsers\PNA-ADMIN\Desktop\Users

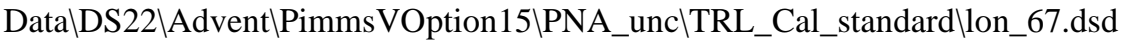

!This file contains the standard definition S-parameters and their uncertainty covariance matrix.

!A comment line begins with the exclamation mark '!'.

!Each frequency corresponds to a line with 74 columns, ordered as below:

!freq(GHz) Nports S11r S11i S12r S12i S21r S21i S22r S22i (continues below)

! V11,11rr V11,11ri V11,11ir V11,11ii V11,12rr V11,12ri V11,12ir V11,12ii V11,21rr V11,21ri V11,21ir V11,21ii V11,22rr V11,22ri V11,22ir V11,22ii (cont.)

! V12,11rr V12,11ri V12,11ir V12,11ii V12,12rr V12,12ri V12,12ir V12,12ii V12,21rr V12,21ri V12,21ir V12,21ii V12,22rr V12,22ri V12,22ir V12,22ii (cont.)

! V21,11rr V21,11ri V21,11ir V21,11ii V21,12rr V21,12ri V21,12ir V21,12ii V21,21rr V21,21ri V21,21ir V21,21ii V21,22rr V21,22ri V21,22ir V21,22ii (cont.)

! V22,11rr V22,11ri V22,11ir V22,11ii V22,12rr V22,12ri V22,12ir V22,12ii V22,21rr V22,21ri V22,21ir V22,21ii V22,22rr V22,22ri V22,22ir V22,22ii (cont.)

!where 'r' stands for real and 'i' for imaginary parts

!One-port standards (Nports $=1$ ) have zeros in 12, 21 and 22 parameters

$0.0500002 .000000-1.417183682230 \mathrm{e}-005-4.443740658109 \mathrm{e}-004 \quad 9.959258901985 \mathrm{e}-001-7.633551774319 \mathrm{e}-$ $002 \quad 9.960169021713 \mathrm{e}-001 \quad-7.633725160536 \mathrm{e}-002 \quad 1.670816517652 \mathrm{e}-003 \quad-3.086126465753 \mathrm{e}-003$ $2.629406670386 \mathrm{e}-005 \quad 4.652891140533 \mathrm{e}-008 \quad 4.652891140533 \mathrm{e}-008 \quad 2.666065297789 \mathrm{e}-005 \quad 4.844344123336 \mathrm{e}-$ $\begin{array}{llllll}0 & -1.726517156037 \mathrm{e}-008 & 5.362774317241 \mathrm{e}-008 & 6.500536557625 \mathrm{e}-007 & 4.839748685965 \mathrm{e}-007 & -\end{array}$ $1.790542883249 \mathrm{e}-008 \quad 5.427044219104 \mathrm{e}-008 \quad 6.496093708699 \mathrm{e}-007-2.490099051392 \mathrm{e}-005 \quad 5.833224653630 \mathrm{e}-$ $\begin{array}{llllll}008 & -5.740619917044 \mathrm{e}-008 & -2.490055849543 \mathrm{e}-005 & 4.844344123336 \mathrm{e}-007 & 5.362774317241 \mathrm{e}-008 & -\end{array}$ $1.726517156037 \mathrm{e}-008 \quad 6.500536557625 \mathrm{e}-007 \quad 5.081731403069 \mathrm{e}-007 \quad 6.264362259717 \mathrm{e}-009 \quad 6.264362259717 \mathrm{e}-$ $009 \quad 6.720691083161 \mathrm{e}-007 \quad 4.852369060167 \mathrm{e}-007 \quad 6.299588023660 \mathrm{e}-009 \quad 6.229418779610 \mathrm{e}-009$ 6.491478513661e-007 2.778003581714e-007 -7.927084406312e-008 9.784160480586e-008 3.849798005501e$\begin{array}{lllll}007 & 4.839748685965 \mathrm{e}-007 & 5.427044219104 \mathrm{e}-008 & -1.790542883249 \mathrm{e}-008 & 6.496093708699 \mathrm{e}-007\end{array}$ $\begin{array}{lllll}4.852369060167 \mathrm{e}-007 & 6.229418779610 \mathrm{e}-009 & 6.299588023660 \mathrm{e}-009 & 6.491478513661 \mathrm{e}-007 & 5.082541031855 \mathrm{e}-\end{array}$ $\begin{array}{llllll}007 & 6.264644517458 \mathrm{e}-009 & 6.264644517458 \mathrm{e}-009 & 6.721800272390 \mathrm{e}-007 & 2.785796988939 \mathrm{e}-007 & -\end{array}$ $\begin{array}{lllll}7.889538374056 \mathrm{e}-008 & 9.746727327217 \mathrm{e}-008 & 3.857689899778 \mathrm{e}-007 & -2.490099051392 \mathrm{e}-005 & -\end{array}$ $5.740619917044 \mathrm{e}-008$ 5.833224653630e-008 -2.490055849543e-005 2.778003581714e-007 9.784160480586e$\begin{array}{llllll}008 & -7.927084406312 \mathrm{e}-008 & 3.849798005501 \mathrm{e}-007 & 2.785796988939 \mathrm{e}-007 & 9.746727327217 \mathrm{e}-008 & -\end{array}$ $\begin{array}{lllll}7.889538374056 \mathrm{e}-008 & 3.857689899778 \mathrm{e}-007 & 2.583763016354 \mathrm{e}-005 & 2.395453001697 \mathrm{e}-008 & 2.395453001697 \mathrm{e}-\end{array}$ $008 \quad 2.610677847297 \mathrm{e}-005$

$0.1000002 .000000 \quad 7.964514736603 \mathrm{e}-004 \quad 1.104855752809 \mathrm{e}-004 \quad 9.862836162465 \mathrm{e}-001-1.552144378366 \mathrm{e}-$ $\begin{array}{lllll}001 & 9.863851361764 \mathrm{e}-001 & -1.551883786862 \mathrm{e}-001 & 2.276911590239 \mathrm{e}-003 & -2.220979434774 \mathrm{e}-003\end{array}$ $2.596062095920 \mathrm{e}-005 \quad 5.399510641220 \mathrm{e}-008 \quad 5.399510641220 \mathrm{e}-008 \quad 2.610561492869 \mathrm{e}-005 \quad 3.106370450985 \mathrm{e}-$ $007 \quad-2.249395253543 \mathrm{e}-009 \quad 4.050125954139 \mathrm{e}-008 \quad 3.885847800197 \mathrm{e}-007 \quad 3.101711514215 \mathrm{e}-007 \quad-$ $2.215188140957 \mathrm{e}-009 \quad 4.046749753322 \mathrm{e}-008 \quad 3.881279903633 \mathrm{e}-007-2.436676501873 \mathrm{e}-005 \quad 5.459365530451 \mathrm{e}-$ $008 \quad-5.602564963107 \mathrm{e}-008 \quad-2.436591832711 \mathrm{e}-005 \quad 3.106370450985 \mathrm{e}-007 \quad 4.050125954139 \mathrm{e}-008 \quad-$ $2.249395253543 \mathrm{e}-009 \quad 3.885847800197 \mathrm{e}-007 \quad 4.009276464479 \mathrm{e}-007 \quad 4.750620570132 \mathrm{e}-009 \quad 4.750620570132 \mathrm{e}-$ $009 \quad 4.635273921334 \mathrm{e}-007 \quad 3.787601214766 \mathrm{e}-007 \quad 4.756674234892 \mathrm{e}-009 \quad 4.742879161361 \mathrm{e}-009$ $4.413662960979 \mathrm{e}-007 \quad 2.219293322771 \mathrm{e}-007-9.246495486997 \mathrm{e}-008$ 9.722296798676e-008 2.526700557380e$\begin{array}{lllll}007 & 3.101711514215 \mathrm{e}-007 & 4.046749753322 \mathrm{e}-008 & -2.215188140957 \mathrm{e}-009 & 3.881279903633 \mathrm{e}-007\end{array}$ 3.787601214766e-007 4.742879161361e-009 4.756674234892e-009 4.413662960979e-007 4.024762146123e$\begin{array}{llllll}007 & 4.748932610921 \mathrm{e}-009 & 4.748932610921 \mathrm{e}-009 & 4.650888187185 \mathrm{e}-007 & 2.225385386834 \mathrm{e}-007 & -\end{array}$ $\begin{array}{lllll}9.248890857054 \mathrm{e}-008 & 9.724610178296 \mathrm{e}-008 & 2.532824230252 \mathrm{e}-007 & -2.436676501873 \mathrm{e}-005 & -\end{array}$ 5.602564963107e-008 5.459365530451e-008 -2.436591832711e-005 2.219293322771e-007 9.722296798676e$\begin{array}{llllll}008 & -9.246495486997 \mathrm{e}-008 & 2.526700557380 \mathrm{e}-007 & 2.225385386834 \mathrm{e}-007 & 9.724610178296 \mathrm{e}-008 & -\end{array}$

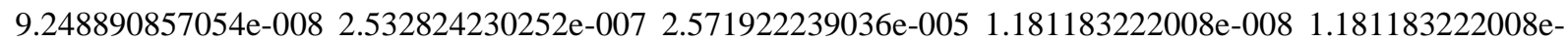
$008 \quad 2.579997842679 \mathrm{e}-005$ 